\title{
Higher Twist Effects In Proton-Proton Collisions
}

\author{
A. I. Ahmadov ${ }^{1,2} *$ I. Boztosun ${ }^{1}$, R. Kh. Muradov ${ }^{2}$, A. Soylu ${ }^{1}$, and E. A. Dadashov ${ }^{2}$ \\ ${ }^{1}$ Department of Physics, Faculty of Arts and Sciences, \\ Erciyes University, Kayseri, Turkey \\ 2 Baku State University, Z. Khalilov st. 23, AZ-1148, Baku, Azerbaijan
}

(Dated: October 14, 2018)

\begin{abstract}
In this article, we investigate the contribution of the high twist Feynman diagrams to the large- $p_{T}$ pion production cross section in proton-proton collisions and we present the general formulae for the high and leading twist differential cross sections. The pion wave function where two non-trivial Gegenbauer coefficients $a_{2}$ and $a_{4}$ have been extracted from the CLEO data, two other pion model wave functions, $P_{2}, P_{3}$, the asymptotic and the Chernyak-Zhitnitsky wave functions are used in the calculations. The results of all the calculations reveal that the high twist cross sections, the ratios $R, r$, the dependence transverse momentum $p_{T}$ and the rapidity $y$ of pion in the $\Phi_{C L E O}\left(x, Q^{2}\right)$ wave function case is very close to the $\Phi_{a s y}(x)$ asymptotic wave function case. It is shown that the high twist contribution to the cross section depends on the choice of the meson wave functions.

PACS numbers: 12.38.-t, 13.60.Le, 14.40.Aq, 13.87.Fh,
\end{abstract}

Keywords: leading twist, high twist, pion wave function

*Electronic address: E-mail:ahmadovazar@yahoo.com

Typeset by REVTEX 


\section{INTRODUCTION}

During the last few years, a great deal of progress has been made in the investigation of the properties of hadronic wave functions[1-12]. The pion wave functions (also called distribution amplitudes -DA) [1] play a key role in the hard-scattering QCD processes because they encapsulate the essential nonperturbative features of the pion's internal structure in terms of the parton's longitudinal momentum fractions $x_{i}$. Meson wave functions have been extensively studied by using QCD sum rules. The original suggestion by Chernyak and Zhitnitsky of a "double-humped" wave function of the pion at a low scale, far from the asymptotic form, was based on an extraction of the first few moments from a standard QCD sum rule approach[5], which has been criticized and revised in Refs.[6,7]. Subsequently, a number of authors have proposed and studied the modified versions of meson $[7,8]$ and baryon wave functions $[9,10]$. Interesting nucleon functions have been constructed in the context of the quark-diquark model[11]. Additional arguments in favour of a form of the pion wave functions close to the asymptotic one have come from the analysis of the transition form factor $\gamma \gamma^{\star} \rightarrow \pi^{0}[12]$. The measurements of this form factor by the CLEO collaboration are consistent with a near-asymptotic form of the wave function[13]. In [14], the leading-twist wave function of the pion at a low normalization point is calculated in the effective low-energy theory derived from the instanton vacuum. These results for the pion wave function at the low normalization point are close to the asymptotic form and consistent with the CLEO measurements. The authors have obtained a shape substantially different from the Chernyak-Zhitnitsky one because they have chosen a significantly smaller value of the second moment, and, more importantly, they have taken all the moments of the wave function into account. Their results support the conclusions reached previously in Refs.[6,7]. The QCD factorization theorems predict that the hadron-hadron cross section can be obtained by the convolution of parton distribution functions and a cross section of the corresponding hard scattering subprocess. The parton distributions are nonperturbative, process-independent quantities, which are specific to any given hadron. The hard scattering cross sections are independent of all long distance effects and can be found by means of pQCD. In the framework of pQCD, the higher order corrections to the hard scattering, and therefore to the hadron-hadron process cross sections, have been calculated [15]. These corrections are large and change the leading order results considerably. Other corrections 
to the hadron-hadron process cross sections and its different characteristics come from the higher twist (HT) terms. Thus, explicit HT effects associated with a meson bound state in the process $\pi N \rightarrow \gamma^{\star} X$ and $\pi N \rightarrow \gamma X$ and their influence on the decay angular distribution have been found by Brodsky and Berger in Ref.[16]. It is important to note that the term "twist" ("twist"- means dimension minus Lorentz spin) is one of the characteristics of composite operators that occur in operator product expansion (OPE) in deep inelastic scattering (DIS). In DIS, the higher twist (twist $>2$ ) terms in OPE are associated with power suppressed corrections. On the other hand, in the various hard hadron-hadron scatteringsi.e. in the Drell-Yan process lacking an (OPE) description- the higher twist terms refer to contributions which are suppressed as $O\left(1 / Q^{2}\right)$, relative to the scale of the hard scatttering. Therefore, in order to calculate the power suppressed corrections to the hadron-hadron collisions cross sections, the Feynman diagram approach should be used. Indeed, in the context of this method, the factorization of the HT contributions of $O\left(1 / Q^{2}\right)$ in hadron collisions has been proven [15]. It is well known that in hadron-hadron scattering at the $O\left(1 / Q^{4}\right)$ level, the factorization fails, which has been demonstrated by the existence of non-cancelling infrared divergences at two loops and by finite terms at one loop[17]. So, the extension of the factorization to $O\left(1 / Q^{2}\right)$ corrections in a large class of hadronic processes helps to place their existing treatments $[16,18]$ on a solid foundation. In [15], the leading $1 / Q^{2}$ corrections to the Drel-Yan cross-section have also been obtained. It is worth noting that the normalization of the $O\left(1 / Q^{2}\right)$ longitudinal structure functions in the Drell-Yan cross-section are determined by higher twist longitudinal structure functions in DIS. In other words, the $1 / Q^{2}$ corrections to the Drell-Yan process can be expressed in terms of the same multiparton correlations as in the DIS one.

On the other hand, the results obtained are consistent with the lowest order calculation of the pion-hadron scattering carried out by Brodsky and Berger in Ref.[16]. By taking these points into account, it may be asserted that the analysis of the higher twist effects on the dependence of the pion wave function in pion production at proton-proton collisions are significant in both theoretical and experimental studies.

Another important aspect of this study is the choice of the meson model wave functions. In this respect, the contribution of the high twist Feynman diagrams to a pion production cross section in proton-proton collisions has been computed by using various pion wave functions. Also, the leading and high twist contributions have been estimated and compared 
to each other. Within this context, this paper is organized as follows: in section II, we provide some formulae for the calculation of the contribution of the high twist diagrams. In section III] we provide the formulae for the calculation of the contribution of the leading twist diagrams and in section IV] we present the numerical results for the cross section and discuss the dependence of the cross section on the pion wave functions. We state our conclusions in section $\nabla$

\section{CONTRIBUTION OF THE HIGH TWIST DIAGRAMS}

The high twist Feynman diagrams, which describe the subprocess $q_{1}+\bar{q}_{2} \rightarrow \pi^{+}\left(\pi^{-}\right)+\gamma$ for the pion production in the proton-proton collision are shown in Fig.1. In the high twist diagrams, the pion of a proton quark is directly observed. Their $1 / Q^{2}$ power suppression is caused by a hard gluon exchange between pion constituents. The amplitude for this subprocess can be found by means of the Brodsky-Lepage formula [19]

$$
M(\hat{s}, \hat{t})=\int_{0}^{1} d x_{1} \int_{0}^{1} d x_{2} \delta\left(1-x_{1}-x_{2}\right) \Phi_{\pi}\left(x_{1}, x_{2}, Q^{2}\right) T_{H}\left(\hat{s}, \hat{t} ; x_{1}, x_{2}\right) .
$$

In Eq.(2.1), $T_{H}$ is the sum of the graphs contributing to the hard-scattering part of the subprocess. The hard-scattering part for the subprocess under consideration is $q_{1}+\bar{q}_{2} \rightarrow$ $\left(q_{1} \bar{q}_{2}\right)+\gamma$, where a quark and antiquark form a pseudoscalar, color-singlet state $\left(q_{1} \bar{q}_{2}\right)$. The hard-scattering amplitude $T_{H}\left(\hat{s}, \hat{t} ; x_{1}, x_{2}\right)$ depends on a process and can be obtained in the framework of pQCD, whereas the wave function $\Phi_{\pi}\left(x_{1}, x_{2}, Q^{2}\right)$ describes all the nonperturbative and process-independent effects of hadronic binding. The hadron wave function gives the amplitude for finding partons (quarks, gluons) carrying the longitudinal fractional momenta $\mathbf{x}=\left(x_{1}, x_{2}, \ldots x_{n}\right)$ and virtualness up to $Q^{2}$ within the hadron and, in general, includes all Fock states with quantum numbers of the hadron. But only the lowest Fock state $\left(q_{1} \bar{q}_{2}\right.$-for mesons, uud-for proton, etc.) contributes to the leading scaling behavior, other Fock state contributions are suppressed by powers of $1 / Q^{2}$. In our work, we have restricted ourselves to considering the lowest Fock state for a meson. Then $\mathbf{x}=x_{1}, x_{2}$ and $x_{1}+x_{2}=1$. This approach can be applied not only to the investigation of exclusive processes, but also to the calculation of higher twist corrections to some inclusive processes. 
The $q_{1} \bar{q}_{2}$ spin state used in computing $T_{H}$ may be written in the form

$$
\sum_{s_{1}, s_{2}} \frac{u_{s_{1}}\left(x_{1} p_{M}\right) \bar{v}_{s_{2}}\left(x_{2} p_{M}\right)}{\sqrt{x_{1}} \sqrt{x_{2}}} \cdot N_{s_{1} s_{2}}^{s}=\left\{\begin{array}{c}
\frac{\gamma_{5} \hat{p}_{\pi}}{\sqrt{2}}, \pi \\
\frac{\hat{p}_{M}}{\sqrt{2}}, \rho_{L} \text { helicity } 0 \\
\mp \frac{\varepsilon_{\mp} \hat{p}_{M}}{\sqrt{2}}, \rho_{T} \text { helicity } \pm 1
\end{array}\right.
$$

where $\varepsilon_{ \pm}=\mp(1 / \sqrt{2})(0,1, \pm i, 0)$ in a frame with $\left(p_{M}\right)_{1,2}=0$ and the $N_{s_{1} s_{2}}^{s}$ project out a state of spins $s$, and $p_{M}$ is the four-momentum of the final meson. In our calculation, we have neglected the pion and the proton masses. Turning to extracting the contributions of the high twist subprocesses, there are many kinds of leading twist subprocesses in $p p$ collisions as the background of the high twist subprocess $q_{1}+q_{2} \rightarrow \pi^{+}\left(\right.$or $\left.\pi^{-}\right)+\gamma$, such as $q+\bar{q} \rightarrow \gamma+g\left(g \rightarrow \pi^{+}\left(\pi^{-}\right)\right), q+g \rightarrow \gamma+q\left(q \rightarrow \pi^{+}\left(\pi^{-}\right)\right), \bar{q}+g \rightarrow \gamma+\bar{q} g\left(\bar{q} \rightarrow \pi^{+}\left(\pi^{-}\right)\right)$and so on. The contributions from these leading twist subprocesses strongly depend on some phenomenological factors, for example, quark and gluon distribution functions in proton and fragmentation functions of various constituents etc. Most of these factors have not been well determined, neither theoretically nor experimentally. Thus they cause very large uncertainty in the computation of the cross section of process $p p \rightarrow \pi^{+}\left(\right.$or $\left.\pi^{-}\right)+\gamma+X$. In general, the magnitude of this uncertainty is much larger than the sum of all the high twist contributions, so it is very difficult to extract the high twist contributions.

The Mandelstam invariant variables for subprocesses $q_{1}+\bar{q}_{2} \rightarrow \pi^{+}\left(\pi^{-}\right)+\gamma$ are defined as

$$
\hat{s}=\left(p_{1}+p_{2}\right)^{2}, \quad \hat{t}=\left(p_{1}-p_{\pi}\right)^{2}, \quad \hat{u}=\left(p_{1}-p_{\gamma}\right)^{2} .
$$

In our calculation, we have also neglected the quark masses. We have aimed to calculate the pion production cross section and to fix the differences due to the use of various pion model functions. We have used five different functions: the asymptotic wave function ASY, the Chernyak-Zhitnitsky $[2,5], P_{2}, P_{3}$ model functions $[7,8]$ and the wave function in which two non-trivial Gegenbauer coefficients $a_{2}$ and $a_{4}$ have been extracted from the CLEO data on the $\gamma \gamma^{\star} \rightarrow \pi^{0}$ transition form factor [20]. In ref.[20], the authors have used the QCD light-cone sum rules approach and have included into their analysis the NLO perturbative and twist-four corrections. They found that in the model with two nonasymptotic terms, at the scale $\mu_{0}=2.4 \mathrm{GeV}$.

$$
\Phi_{a s y}(x)=\sqrt{3} f_{\pi} x(1-x), \quad \Phi_{C Z}\left(x, \mu_{0}^{2}\right)=5 \Phi_{a s y}(2 x-1)^{2},
$$




$$
\begin{gathered}
\Phi_{P_{2}}\left(x, \mu_{0}^{2}\right)=\Phi_{a s y}(x)\left[-0.1821+5.91(2 x-1)^{2}\right] \\
\Phi_{P_{3}}\left(x, \mu_{0}^{2}\right)=\Phi_{a s y}(x)\left[0.6016-4.659(2 x-1)^{2}+15.52(2 x-1)^{4}\right], \\
\Phi_{C L E O}\left(x, \mu_{0}^{2}\right)=\Phi_{a s y}(x)\left[1+0.19 C_{2}^{3 / 2}(2 x-1)-0.14 C_{4}^{3 / 2}(2 x-1)\right],
\end{gathered}
$$

where $f_{\pi}=0.0923 \mathrm{GeV}$ is the pion decay constant. Here, we have denoted by $x \equiv x_{1}$, the longitudinal fractional momentum carried by the quark within the meson. Then, $x_{2}=1-x$ and $x_{1}-x_{2}=2 x-1$. The pion wave function is symmetric under replacement $x_{1}-x_{2} \leftrightarrow$ $x_{2}-x_{1}$. The values of the pion wave function moments $<\xi^{n}>$ are defined as

$$
<\xi^{n}>=\int_{-1}^{1} d \xi \xi^{n} \widetilde{\Phi}_{\pi}(\xi)
$$

Here, $\widetilde{\Phi}_{\pi}(\xi)$ is the model function without $f_{\pi}$ and $\xi=x_{1}-x_{2}$. The pion wave function moments have been calculated by means of the QCD sum rules method by Chernyak and Zhitnitsky at the normalization point $\mu_{0}=0.5 \mathrm{GeV}$. They are equal to

$$
<\xi^{0}>_{\mu_{0}}=1,<\xi^{2}>_{\mu_{0}}=0.44,<\xi^{4}>_{\mu_{0}}=0.27
$$

The Chernyak-Zhitnitsky pion model wave function has the following moments

$$
<\xi^{0}>_{\mu_{0}}=1,<\xi^{2}>_{\mu_{0}}=0.43,<\xi^{4}>_{\mu_{0}}=0.24
$$

It is interesting to note that the corresponding moments of the asymptotic wave function differ considerably from those in Eqs.(2.6), (2.7)

$$
<\xi^{0}>_{\mu_{0}}=1,<\xi^{2}>_{\mu_{0}}=0.20,<\xi^{4}>_{\mu_{0}}=0.086
$$

This means that the realistic pion wave function is much wider than the asymptotic one [5,21]. We have also used two other functions $P_{2}$ and $P_{3}$. The wave function $P_{2}$ is a quadratic polynomial. Its free parameter, which is defined as the ratio of the coefficients of the constant and quadratic terms, is fixed to give the best fit to the values of the 2nd and 4th moments of the wave function. The model function $P_{2}$ has the same shape as the $C Z$ one, the difference being in the constant term. The function $P_{3}$ is a polynomial in which the number of free parameters is equal to the number of independent moments. These parameters are completely fixed by the same moment of the pion wave function. $P_{3}$ has a form different from that of the $C Z$ and $P_{2}$ ones. All these wave functions, of course, have 
the same zeroth moment by construction. Thus, the comparison of the predictions obtained by using the $C Z$ and $P_{2}$ ones with the ones obtained by means of $P_{3}$ enables us to determine the sensitivity of the predictions to the form of the wave function.

The model functions can be written as

$$
\begin{gathered}
\Phi_{a s y}(x)=\sqrt{3} f_{\pi} x(1-x), \\
\Phi_{C Z}\left(x, \mu_{0}^{2}\right)=\Phi_{a s y}(x)\left[C_{0}^{3 / 2}(2 x-1)+\frac{2}{3} C_{2}^{3 / 2}(2 x-1)\right] \\
\Phi_{P_{2}}\left(x, \mu_{0}^{2}\right)=\Phi_{a s y}(x)\left[C_{0}^{3 / 2}(2 x-1)+0.788 C_{2}^{3 / 2}(2 x-1)\right], \\
\Phi_{P_{3}}\left(x, \mu_{0}^{2}\right)=\Phi_{a s y}(x)\left[C_{0}^{3 / 2}(2 x-1)+0.7584 C_{2}^{3 / 2}(2 x-1)+0.3942 C_{4}^{3 / 2}(2 x-1)\right], \\
\Phi_{C L E O}\left(x, \mu_{0}^{2}\right)=\Phi_{a s y}(x)\left[C_{0}^{3 / 2}(2 x-1)+0.19 C_{2}^{3 / 2}(2 x-1)-0.14 C_{4}^{3 / 2}(2 x-1)\right], \\
C_{0}^{3 / 2}(2 x-1)=1, C_{2}^{3 / 2}(2 x-1)=\frac{3}{2}\left(5(2 x-1)^{2}-1\right), \\
C_{4}^{3 / 2}(2 x-1)=\frac{15}{8}\left(21(2 x-1)^{4}-14(2 x-1)^{2}+1\right) .
\end{gathered}
$$

It may be seen that the pion wave function extracted from the experimental data depends on the methods used and their accuracy. Although one may claim that the meson wave function is a process-independent quantity, describing the internal structure of the meson itself, the exploration of different exclusive processes with the same meson leads to a variety of wave functions. This means that the methods employed have shortcomings or do not encompass all the mechanisms important for a given process. Such a situation is pronounced in the case of the pion. It is known that the pion wave function (distribution amplitude-DA) can be expanded over the eigenfunctions of the one-loop Brodsky-Lepage equation, i.e., in terms of the Gegenbauer polynomials $\left\{C_{n}^{3 / 2}(2 x-1)\right\}$,

$$
\Phi_{\pi}\left(x, Q^{2}\right)=\Phi_{a s y}(x)\left[1+\sum_{n=2,4 . .}^{\infty} a_{n}\left(Q^{2}\right) C_{n}^{3 / 2}(2 x-1)\right],
$$

The evolution of the wave function (DA) on the factorization scale $Q^{2}$ is governed by the functions $a_{n}\left(Q^{2}\right)$,

$$
\begin{gathered}
a_{n}\left(Q^{2}\right)=a_{n}\left(\mu_{0}^{2}\right)\left[\frac{\alpha_{s}\left(Q^{2}\right)}{\alpha_{s}\left(\mu_{0}^{2}\right)}\right]^{\gamma_{n} / \beta_{0}}, \\
\frac{\gamma_{2}}{\beta_{0}}=\frac{50}{81}, \frac{\gamma_{4}}{\beta_{0}}=\frac{364}{405}, n_{f}=3 .
\end{gathered}
$$


In Eq.(2.11), $\left\{\gamma_{n}\right\}$ are anomalous dimensions defined by the expression,

$$
\gamma_{n}=C_{F}\left[1-\frac{2}{(n+1)(n+2)}+4 \sum_{j=2}^{n+1} \frac{1}{j}\right] .
$$

The constants $a_{n}\left(\mu_{0}^{2}\right)=a_{n}^{0}$ are input parameters that form the shape of the wave functions and which can be extracted from experimental data or obtained from the nonperturbative QCD computations at the normalization point $\mu_{0}^{2}$. The QCD coupling constant $\alpha_{s}\left(Q^{2}\right)$ at the two-loop approximation is given by the expression

$$
\alpha_{s}\left(Q^{2}\right)=\frac{4 \pi}{\beta_{0} \ln \left(Q^{2} / \Lambda^{2}\right)}\left[1-\frac{2 \beta_{1}}{\beta_{0}^{2}} \frac{\ln \ln \left(Q^{2} / \Lambda^{2}\right)}{\ln \left(Q^{2} / \Lambda^{2}\right)}\right] .
$$

Here, $\Lambda$ is the QCD scale parameter, $\beta_{0}$ and $\beta_{1}$ are the QCD beta function one- and two-loop coefficients, respectively,

$$
\beta_{0}=11-\frac{2}{3} n_{f}, \quad \beta_{1}=51-\frac{19}{3} n_{f} .
$$

The cross section for the high twist subprocess is given by the expression

$$
\frac{d \sigma}{d \hat{t}}(\hat{s}, \hat{t}, \hat{u})=\frac{8 \pi^{2} \alpha_{E} C_{F}}{27} \frac{[D(\hat{t}, \hat{u})]^{2}}{\hat{s}^{3}}\left[\frac{1}{\hat{u}^{2}}+\frac{1}{\hat{t}^{2}}\right]
$$

where

$$
D(\hat{t}, \hat{u})=e_{1} \hat{t} \int_{0}^{1} d x_{1}\left[\frac{\alpha_{s}\left(Q_{1}^{2}\right) \Phi_{\pi}\left(x_{1}, Q_{1}^{2}\right)}{1-x_{1}}\right]+e_{2} \hat{u} \int_{0}^{1} d x_{1}\left[\frac{\alpha_{s}\left(Q_{2}^{2}\right) \Phi_{\pi}\left(x_{1}, Q_{2}^{2}\right)}{1-x_{1}}\right],
$$

where $Q_{1}^{2}=\left(x_{1}-1\right) \hat{u}, \quad Q_{2}^{2}=-x_{1} \hat{t}$, represents the momentum squared carried by the hard gluon in Fig.1, $e_{1}\left(e_{2}\right)$ is the charge of $q_{1}\left(\bar{q}_{2}\right)$ and $C_{F}=\frac{4}{3}$. The high twist contribution to the large- $p_{T}$ pion production cross section in the process $p p \rightarrow \pi^{+}\left(\pi^{-}\right)+\gamma$ is $[22-24]$

$$
\begin{gathered}
\Sigma_{M}^{H T} \equiv E \frac{d \sigma}{d^{3} p}=\int_{0}^{1} \int_{0}^{1} d x_{1} d x_{2} G_{q_{1} / h_{1}}\left(x_{1}\right) G_{q_{2} / h_{2}}\left(x_{2}\right) \frac{\hat{s}}{\pi} \frac{d \sigma}{d \hat{t}}(q \bar{q} \rightarrow \pi \gamma) \delta(\hat{s}+\hat{t}+\hat{u}) \\
\pi E \frac{d \sigma}{d^{3} p}=\frac{d \sigma}{d y d p_{T}^{2}} \\
\hat{s}=x_{1} x_{2} s \\
\hat{t}=x_{1} t \\
\hat{u}=x_{2} u \\
t=-m_{T} \sqrt{s} e^{-y}=-p_{T} \sqrt{s} e^{-y}
\end{gathered}
$$




$$
\begin{gathered}
u=-m_{T} \sqrt{s} e^{y}=-p_{T} \sqrt{s} e^{y}, \\
x_{1}=-\frac{x_{2} u}{x_{2} s+t}=\frac{x_{2} p_{T} \sqrt{s} e^{y}}{x_{2} s-p_{T} \sqrt{s} e^{-y}}, \\
x_{2}=-\frac{x_{1} t}{x_{1} s+u}=\frac{x_{1} p_{T} \sqrt{s} e^{-y}}{x_{1} s-p_{T} \sqrt{s} e^{y}},
\end{gathered}
$$

where $m_{T}$ - is the transverse mass of pion, which is given by

$$
m_{T}^{2}=m^{2}+p_{T}^{2}
$$

For a full discussion, we consider a difference $\Delta^{H T}$ between the high twist cross section combinations $\Sigma_{\pi^{+}}^{H T}$ and $\Sigma_{\pi^{-}}^{H T}$

$$
\Delta_{\pi}^{H T}=\Sigma_{\pi^{+}}^{H T}-\Sigma_{\pi^{-}}^{H T}=E_{\pi^{+}} \frac{d \sigma}{d^{3} p}\left(p p \rightarrow \pi^{+} \gamma\right)-E_{\pi^{-}} \frac{d \sigma}{d^{3} p}\left(p p \rightarrow \pi^{-} \gamma\right)
$$

We have extracted the following high twist subprocesses contributing to the two covariant cross sections in Eq.(2.16)

$$
\frac{d \sigma^{1}}{d \hat{t}}\left(u \bar{d} \rightarrow \pi^{+} \gamma\right), \quad \frac{d \sigma^{2}}{d \hat{t}}\left(\bar{d} u \rightarrow \pi^{+} \gamma\right), \quad \frac{d \sigma^{3}}{d \hat{t}}\left(\bar{u} d \rightarrow \pi^{-} \gamma\right), \quad \frac{d \sigma^{4}}{d \hat{t}}\left(d \bar{u} \rightarrow \pi^{-} \gamma\right),
$$

By charge conjugation invariance, we have

$$
\frac{d \sigma^{1}}{d \hat{t}}\left(u \bar{d} \rightarrow \pi^{+} \gamma\right)=\frac{d \sigma^{3}}{d \hat{t}}\left(\bar{u} d \rightarrow \pi^{-} \gamma\right), \text { and } \frac{d \sigma^{2}}{d \hat{t}}\left(\bar{d} u \rightarrow \pi^{+} \gamma\right)=\frac{d \sigma^{4}}{d \hat{t}}\left(d \bar{u} \rightarrow \pi^{-} \gamma\right)
$$

\section{CONTRIBUTION OF THE LEADING TWIST DIAGRAMS}

Regarding the high twist corrections to the pion production cross section, a comparison of our results with leading twist contributions is crucial. The leading twist subprocesses for the pion production are quark-antiquark annihilation $q \bar{q} \rightarrow g \gamma, g \rightarrow \pi^{+}\left(\pi^{-}\right)$, shown in Fig.2. The corresponding cross section is easily verified as[22]

$$
\frac{d \sigma}{d \hat{t}}(q \bar{q} \rightarrow g q)=\frac{8}{9} \pi \alpha_{E} \alpha_{s}\left(Q^{2}\right) \frac{e_{q}^{2}}{\hat{s}^{2}}\left(\frac{\hat{t}}{\hat{u}}+\frac{\hat{u}}{\hat{t}}\right)
$$

For the leading-twist contribution, we find

$$
\Sigma_{M}^{L T} \equiv E \frac{d \sigma}{d^{3} p}=\sum_{q} \int_{0}^{1} d x_{1} d x_{2} d z G_{q_{1} / h_{1}}\left(x_{1}\right) G_{q_{2} / h_{2}}\left(x_{2}\right) D_{g}^{\pi}(z) \frac{\hat{s}}{\pi z^{2}} \frac{d \sigma}{d \hat{t}}(q \bar{q} \rightarrow g \gamma) \delta(\hat{s}+\hat{t}+\hat{u}),
$$

where

$$
\hat{s}=x_{1} x_{2} s, \hat{t}=\frac{x_{1} t}{z}, \hat{u}=\frac{x_{2} u}{z}, z=-\frac{x_{1} t+x_{2} u}{x_{1} x_{2} s} .
$$


$D_{g}^{\pi}(z)=D_{g}^{\pi^{+}}(z)=D_{g}^{\pi^{-}}(z)$ represents the gluon fragmentation function into a meson containing a gluon of the same flavor. In the leading twist subprocess, $\pi$ meson is indirectly emitted from the gluon with fractional momentum $z$. The $\delta$ function may be expressed in terms of the parton kinematic variables, and the $z$ integration may then be done. The final form for the cross section is

$$
\begin{gathered}
\Sigma_{M}^{L T} \equiv E \frac{d \sigma}{d^{3} p}=\sum_{q} \int_{x_{1 m i n}}^{1} d x_{1} \int_{x_{2 m i n}}^{1} d x_{2} G_{q_{1} / h_{1}}\left(x_{1}\right) G_{q_{2} / h_{2}}\left(x_{2}\right) D_{g}^{\pi}(z) \times \\
\frac{1}{\pi z} \frac{d \sigma}{d \hat{t}}(q \bar{q} \rightarrow g \gamma)=\sum_{q} \int_{x_{1 m i n}}^{1} d x_{1} \int_{x_{2 m i n}}^{1} d x_{2} \frac{x_{1} G_{q_{1} / h_{1}}\left(x_{1}\right) s x_{2} G_{q_{2} / h_{2}}\left(x_{2}\right)}{-\left(x_{1} t+x_{2} u\right)} \frac{D_{g}^{\pi}(z)}{\pi} \frac{d \sigma}{d \hat{t}}(q \bar{q} \rightarrow g \gamma) .
\end{gathered}
$$

\section{NUMERICAL RESULTS AND DISCUSSION}

In this section, the numerical results for higher twist effects on the dependence of the chosen meson wave functions in the process $p p \rightarrow \pi^{+}\left(\right.$or $\left.\pi^{-}\right) \gamma$ are discussed. We have calculated the dependence on the pion wave functions for the high twist contribution to the large- $p_{T}$ pion production cross section in the proton-proton collision. In the calculations, the asymptotic $\Phi_{a s y}$, Chernyak-Zhitnitsky $\Phi_{C Z}$, two other pion model functions, $\Phi_{P_{2}}, \Phi_{P_{3}}$ and also, the pion wave function, from which two non-trivial Gegenbauer coefficients $a_{2}$ and $a_{4}$ have been extracted from the CLEO data on the $\pi^{0} \gamma$ transition form factor have been used[20]. In the ref.[20], authors have used the QCD light-cone sum rules approach and included into their analysis the NLO perturbative and twist-four corrections. For the high twist subprocess, we take $q_{1}+\bar{q}_{2} \rightarrow\left(q_{1} \bar{q}_{2}\right)+\gamma$ and we have extracted the following four high twist subprocess $u \bar{d} \rightarrow \pi^{+} \gamma, \bar{d} u \rightarrow \pi^{+} \gamma, \bar{u} d \rightarrow \pi^{-} \gamma, d \bar{u} \rightarrow \pi^{-} \gamma$ contributing to $p p \rightarrow \pi^{+}$(or $\left.\pi^{-}\right) \gamma$ cross sections. For the dominant leading twist subprocess for the pion production, we take the quark-antiquark annihilation $q \bar{q} \rightarrow g \gamma$, in which the $\pi$ meson is indirectly emitted from the gluon. As an example for the quark distribution function inside the proton, the MRST2003c package [25] has been used. The gluon fragmentation function has been taken from [26]. The other problems dealth with are the choice of the QCD scale parameter $\Lambda$ and the number of the active quark flavors $n_{f}$. The high twist subprocesses probe the meson wave functions over a large range of $Q^{2}$ squared momentum transfer, carried 
by the gluon. Therefore, phenomenologically, in the given diagram in Fig 1, for the centerof-mass energy $\sqrt{s}=63 \mathrm{GeV}$, we take $Q^{2}=p_{T}^{2}$. However, for the center-of-mass energy $\sqrt{s}=630 \mathrm{GeV}$, we take $Q_{1}^{2}=\left(x_{1}-1\right) \hat{u}, Q_{2}^{2}=-x_{1} \hat{t}$, which we have obtained directly from the high twist subprocesses diagrams. The same $Q^{2}$ has been used as an argument of $\alpha_{s}\left(Q^{2}\right)$ in the calculation of each diagram. The results of our numerical calculations are plotted in Figs.3-18. Figs.3-5 show the dependence of the differential cross sections of the high twist $\Sigma_{\pi^{+}}^{H T}$, leading twist plus high twist $\Sigma_{\pi^{+}}^{t o t}=\Sigma_{\pi^{+}}^{L T}+\Sigma_{\pi^{+}}^{H T}$ and ratio $R=\Sigma_{\pi^{+}}^{H T} / \Sigma_{\pi^{+}}^{L T}$ as a function of the pion transverse momentum $p_{T}$ for five different meson wave functions. As shown in Figs.3-4, the high twist differential cross section is monotonically decreasing with an increase in the transverse momentum of the pion. As seen from Figs.3-4, in all wave functions of the mesons, the dependencies of the high twist cross sections on the $p_{T}$ transverse momentum of the pion demonstrate the same behavior. On the other hand, the higher twist corrections are very sensitive to the choice of the pion wave function. We should note that the magnitude of the high twist cross section in the pion wave function $\Phi_{C L E O}\left(x, Q^{2}\right)$ case is very close to the asymptotic wave function $\Phi_{a s y}(x)$ case. In Fig.5, the ratio $R=\Sigma_{\pi^{+}}^{H T} / \Sigma_{\pi^{+}}^{L T}$ is plotted at $y=0$ as a function of the pion transverse momentum $p_{T}$ for the different pion wave functions. First of all, it is seen that the values of $R$ for fixed $y$ and $\sqrt{s}$ depend on the choice of the pion wave function. Also, the distinction between $R\left(\Phi_{\text {asy }}(x)\right)$ with $R\left(\Phi_{C L E O}\left(x, Q^{2}\right)\right), R\left(\Phi_{C Z}\left(x, Q^{2}\right)\right), R\left(\Phi_{P_{2}}\left(x, Q^{2}\right)\right)$ and $R\left(\Phi_{P_{3}}\left(x, Q^{2}\right)\right)$ have been calculated. We have found that the distinction $R\left(\Phi_{a s y}(x)\right)$ and $R\left(\Phi_{C L E O}\left(x, Q^{2}\right)\right)$ is small, whereas a distinction between $R\left(\Phi_{a s y}(x)\right)$ with $R\left(\Phi_{C Z}\left(x, Q^{2}\right)\right), R\left(\Phi_{P_{2}}\left(x, Q^{2}\right)\right)$ and $R\left(\Phi_{P_{3}}\left(x, Q^{2}\right)\right)$ is significant. For example, in the case of $\sqrt{s}=63 \mathrm{GeV}, y=0$, the distinction between $R\left(\Phi_{a s y}(x)\right)$ with $R\left(\Phi_{i}\left(x, Q^{2}\right)\right)\left(i=C L E O, C Z, P_{2}, P_{3}\right)$ is shown in Table 【

Thus, the distinction between $R\left(\Phi_{a s y}(x)\right)$ and $R\left(\Phi_{C L E O}\left(x, Q^{2}\right)\right)$ is maximum at $p_{T}=$ $6 G e V / c$, but the distinction between $R\left(\Phi_{\text {asy }}(x)\right)$ with $R\left(\Phi_{C Z}\left(x, Q^{2}\right)\right), R\left(\Phi_{P_{2}}\left(x, Q^{2}\right)\right)$, $R\left(\Phi_{P_{3}}\left(x, Q^{2}\right)\right)$ is maximum at $p_{T}=2 G e V / c$ and decreases with an increase in $p_{T}$. Such a behavior of $R$ may be explained by reducing all moments of the pion model wave functions to those of $\Phi_{a s y}(x)$ for high $Q^{2}$. In Fig.6, we show the difference of the $\Delta_{\pi}^{H T}=\Sigma_{\pi^{+}}^{H T}-\Sigma_{\pi^{-}}^{H T}$ high twist cross section as a function of the pion transverse momentum $p_{T}$ for five different pion wave functions. As seen from Fig.6, when the transverse momentum of the pion is increasing, the difference $\Delta_{\pi}^{H T}$ high twist cross section is monotonically decreasing. As shown in Fig.6, the difference $\Delta_{\pi}^{H T}$ high twist cross section for pion wave function $\Phi_{C L E O}\left(x, Q^{2}\right)$ 
is very close to the asymptotic wave function $\Phi_{a s y}(x)$. In Fig.7, we show the difference $\Delta_{\pi}^{L T}=\Sigma_{\pi^{+}}^{L T} \Sigma_{\pi^{-}}^{L T}$ leading twist and sum of differences leading and high twist $\Delta_{\pi}^{t o t}=\Delta_{\pi}^{L T}+\Delta_{\pi}^{H T}$ cross section for five pion wave functions as a function of the pion transverse momentum $p_{T}$. As in Fig.6, the $\Delta_{\pi}^{L T}$ and $\Delta_{\pi}^{t o t}$ are monotonically decreasing when the transverse momentum $p_{T}$ of the pion is increasing. But, the distinction between the difference $\Delta_{\pi}^{L T}$ leading and $\Delta_{\pi}^{t o t}=\Delta_{\pi}^{L T}+\Delta_{\pi}^{H T}$ sum of the difference leading and the high twist cross section is not evident. In Fig.8, the ratio $r=\Delta_{\pi}^{H T} / \Delta_{\pi}^{L T}$ is plotted at $y=0$ as a function of the pion transverse momentum $p_{T}$ for five pion wave functions. As shown in Fig.8, the values of $r$ for fixed $y$ and $\sqrt{s}$ depend on the choice of pion wave function as in Fig.5. Also, we have calculated the distinction between $r\left(\Phi_{a s y}(x)\right)$ with $r\left(\Phi_{C L E O}\left(x, Q^{2}\right)\right), r\left(\Phi_{C Z}\left(x, Q^{2}\right)\right), r\left(\Phi_{P_{2}}\left(x, Q^{2}\right)\right)$ and $r\left(\Phi_{P_{3}}\left(x, Q^{2}\right)\right)$. For example, in the case of $\sqrt{s}=63 \mathrm{GeV}, y=0$ the distinction between $r\left(\Phi_{\text {asy }}(x)\right)$ with $r\left(\Phi_{i}\left(x, Q^{2}\right)\right)\left(\mathrm{i}=\mathrm{CLEO}, \mathrm{CZ}, P_{2}, P_{3}\right)$ is presented in Table II

We have obtained very interesting results. The calculations show that the ratio $R\left(\Phi_{i}\left(x, Q^{2}\right)\right) / R\left(\Phi_{a s y}(x)\right), \quad\left(\mathrm{i}=\mathrm{CLEO}, \mathrm{CZ}, P_{2}, P_{3}\right)$ of all the transverse momentum $p_{T}$ of the pion is equal to the ratio $r\left(\Phi_{i}\left(x, Q^{2}\right) / r\left(\Phi_{a s y}(x)\right)\right.$, (i=CLEO, CZ, $\left.P_{2}, P_{3}\right)$. In Figs.9-10, we have depicted $\Sigma_{\pi^{+}}^{H T}$ and $\Delta_{\pi}^{H T}=\Sigma_{\pi^{+}}^{H T}-\Sigma_{\pi^{-}}^{H T}$ as a function of the rapidity $y$ of the pion at $\sqrt{s}=63 \mathrm{GeV}$ and $p_{T}=5 \mathrm{GeV} / \mathrm{c}$. As we are now in the high energy region, the change of the rapidity to determine these relations is given by $-\ln \left(\sqrt{s} / p_{T}\right) \leq y \leq \ln \left(\sqrt{s} / p_{T}\right)$. At $\sqrt{s}=63 \mathrm{GeV}$ and $p_{T}=5 \mathrm{GeV} / \mathrm{c}$, the pion rapidity lies in the region $-2.52 \leq y \leq 2.52$. As seen from Figs.9-10, in the region $(-2.52 \leq y \leq 0.85)$, the high twist cross section for asymptotic $\Phi_{a s y}(x)$ and $\Phi_{C L E O}\left(x, Q^{2}\right)$ wave functions increase and it has a maximum approximately at one point $y=0.85$. After that, the cross sections monotonically decrease with an increase in the $y$ rapidity of the pion. As seen from Figs.9-10, the high twist $\Sigma_{\pi^{+}}^{H T}$ and difference high twist $\Delta_{\pi}^{H T}$ cross sections are very sensitive to the choice of meson wave functions. Also, as shown in Figs.9-10, in the regions $-2.52 \leq y \leq-1$ and $1 \leq y \leq 2.52$, the high twist cross section for the $\Phi_{C L E O}\left(x, Q^{2}\right)$ is very close to the $\Phi_{a s y}(x)$ wave function case. We have also carried out comparative calculations in the center-of-mass energy $\sqrt{s}=630 \mathrm{GeV}$. Figs.11-13 show the dependence of the differential cross sections of the high twist $\Sigma_{\pi^{+}}^{H T}$, leading twist plus high twist $\Sigma_{\pi^{+}}^{t o t}=\Sigma_{\pi^{+}}^{L T}+\Sigma_{\pi^{+}}^{H T}$ and ratio $R=\Sigma_{\pi^{+}}^{H T} / \Sigma_{\pi^{+}}^{L T}$ as a function of the pion transverse momentum $p_{T}$ for five different meson wave functions. As shown in Figs.11-12, the high twist differential cross section is monotonically decreasing when the transverse momentum of the pion is increasing. As seen from Figs.11-12, 
for all wave functions of mesons, the dependencies of the high twist cross sections on the transverse momentum of the pion demonstrate the same behavior. But, the higher twist corrections are very sensitive to the choice of the pion wave function. We should note that the magnitude of the high twist cross section for the pion wave function $\Phi_{C L E O}\left(x, Q^{2}\right)$ is very close to the asymptotic wave function $\Phi_{\text {asy }}(x)$ case. In Fig.13, the ratio $R=\Sigma_{\pi^{+}}^{H T} / \Sigma_{\pi^{+}}^{L T}$ is plotted at $y=0$ as a function of the pion transverse momentum $p_{T}$ for the different pion wave functions. First of all, it may be seen that the values of $R$ for fixed $y$ and $\sqrt{s}$ depend on the choice of the pion wave function. As in center-of-mass energy $\sqrt{s}=63 \mathrm{GeV}$, we have also calculated the difference between $R\left(\Phi_{a s y}(x)\right)$ and four other wave functions i.e. $R\left(\Phi_{C L E O}\left(x, Q^{2}\right)\right), R\left(\Phi_{C Z}\left(x, Q^{2}\right)\right), R\left(\Phi_{P_{2}}\left(x, Q^{2}\right)\right)$, and $R\left(\Phi_{P_{3}}\left(x, Q^{2}\right)\right)$. We have found that the distinction between $R\left(\Phi_{\text {asy }}(x)\right)$ and $R\left(\Phi_{C L E O}\left(x, Q^{2}\right)\right)$ is very small, whereas the distinction between $R\left(\Phi_{a s y}(x)\right)$ and $R\left(\Phi_{C Z}\left(x, Q^{2}\right)\right), R\left(\Phi_{P_{2}}\left(x, Q^{2}\right)\right)$ and $R\left(\Phi_{P_{3}}\left(x, Q^{2}\right)\right)$ is significant. In order to demonstrate this, in the case of $\sqrt{s}=630 \mathrm{GeV}, y=0$ the distinction between $R\left(\Phi_{\text {asy }}(x)\right)$ with $R\left(\Phi_{i}\left(x, Q^{2}\right)\right.$ ) (i=CLEO, CZ, $\left.P_{2}, P_{3}\right)$ is shown in Table III.

Thus, the distinction between $R\left(\Phi_{a s y}(x)\right)$ and $R\left(\Phi_{C L E O}\left(x, Q^{2}\right)\right)$ is maximum at $p_{T}=$ $60 \mathrm{GeV} / \mathrm{c}$, but the distinction between $R\left(\Phi_{\text {asy }}(x)\right)$ with $R\left(\Phi_{C Z}\left(x, Q^{2}\right)\right), R\left(\Phi_{P_{2}}\left(x, Q^{2}\right)\right)$, $R\left(\Phi_{P_{3}}\left(x, Q^{2}\right)\right)$ is maximum at $p_{T}=20 \mathrm{GeV} / \mathrm{c}$ and decreases with an increase in $p_{T}$. In Fig.14, we have shown the difference $\Delta_{\pi}^{H T}=\Sigma_{\pi^{+}}^{H T}-\Sigma_{\pi^{-}}^{H T}$ high twist cross section as a function of the pion transverse momentum $p_{T}$, for five different pion wave functions. As seen from Fig.14, as the transverse momentum of pion, $p_{T}$, increases, the difference of the high twist cross section, $\Delta_{\pi}^{H T}$, monotonically decreases. As shown in Fig.14, $\Delta_{\pi}^{H T}$ for the $\Phi_{C L E O}\left(x, Q^{2}\right)$ pion wave function is very close to the asymptotic wave function, $\Phi_{a s y}(x)$. In Fig.15, we have shown $\Delta_{\pi}^{L T}=\Sigma_{\pi^{+}}^{L T}-\Sigma_{\pi^{-}}^{L T} i . e$. the leading twist cross section, and $\Delta_{\pi}^{t o t}=\Delta_{\pi}^{L T}+\Delta_{\pi}^{H T} i . e$. the sum of the leading and the high twist cross sections, for five pion wave functions, as a function of the pion transverse momentum, $p_{T}$. As in Fig.7, the $\Delta_{\pi}^{L T}$ and $\Delta_{\pi}^{t o t}$ cross sections monotonically decrease when the transverse momentum $p_{T}$ of the pion increases. But the distinction between $\Delta_{\pi}^{L T}$ and $\Delta_{\pi}^{t o t}=\Delta_{\pi}^{L T}+\Delta_{\pi}^{H T}$ is not evident. In Fig.16, the ratio $r=\Delta_{\pi}^{H T} / \Delta_{\pi}^{L T}$ is plotted at $y=0$ as a function of the pion transverse momentum, $p_{T}$, for five pion wave functions. As shown in Fig.16, the values of $r$ for fixed $y$ and $\sqrt{s}$ depend on the choice of pion wave function similar to Fig.8. We have also calculated the distinction between $r\left(\Phi_{a s y}\right)$ and $r\left(\Phi_{C L E O}\left(x, Q^{2}\right), r\left(\Phi_{C Z}\left(x, Q^{2}\right)\right), r\left(\Phi_{P_{2}}\left(x, Q^{2}\right)\right.\right.$ and $r\left(\Phi_{P_{3}}\left(x, Q^{2}\right)\right.$. As an example, in the case of $\sqrt{s}=630 \mathrm{GeV}, y=0$ the distinction between $r\left(\Phi_{a s y}(x)\right)$ and $r\left(\Phi_{i}\left(x, Q^{2}\right)\right)$ (i=CLEO, 
$\left.\mathrm{CZ}, P_{2}, P_{3}\right)$ is shown in Table IV.

As seen from calculations with increasing center-of-mass energy from $\sqrt{s}=63 \mathrm{GeV}$ to $\sqrt{s}=630 \mathrm{GeV}$, the distinction between $R$ and $r$ decreases for all pion wave functions. In Figs.17-18, we have depicted $\Sigma_{\pi^{+}}^{H T}$ and $\Delta_{\pi}^{H T}=\Sigma_{\pi^{+}}^{H T} \Sigma_{\pi^{-}}^{H T}$ as a function of the rapidity $y$ of pion at the $\sqrt{s}=630 \mathrm{GeV}$ and $p_{T}=50 \mathrm{GeV} / \mathrm{c}$. Since we are now in the high energy region, the change of the rapidity to determine these relations is $-\ln \left(\sqrt{s} / p_{T}\right) \leq y \leq \ln \left(\sqrt{s} / p_{T}\right)$. At $\sqrt{s}=630 \mathrm{GeV}$ and $p_{T}=50 \mathrm{GeV} / \mathrm{c}$ the pion rapidity also lies in the region $-2.52 \leq y \leq 2.52$. As seen from Figs.17-18, in the region $(-2.52 \leq y \leq 0.85)$, the high twist cross section for the asymptotic $\Phi_{a s y}(x)$ and $\Phi_{C L E O}\left(x, Q^{2}\right)$ wave functions increases and it has a maximum approximately at one point $y=0.85$. After that, the cross sections monotonically decrease with an increase in the $y$ rapidity of the pion. As in Figs.9-10, in the regions $-2.52 \leq y \leq-1$ and $1 \leq y \leq 2.52$, the high twist cross section for $\Phi_{C L E O}\left(x, Q^{2}\right)$ is very close to the $\Phi_{a s y}(x)$ wave function.

\section{CONCLUDING REMARKS}

In this work, we have calculated the higher twist contribution to the large- $p_{T}$ pion production cross section to show the dependence on the chosen meson wave functions in the process $p p \rightarrow \pi^{+}\left(\right.$or $\left.\pi^{-}\right) \gamma$. In our calculations, we have used the asymptotic $\Phi_{a s y}$, ChernyakZhitnitsky $\Phi_{C Z}$, two other pion model functions, $\Phi_{P_{2}}, \Phi_{P_{3}}$ and also, the pion wave function, in which the coefficients $a_{2}$ and $a_{4}$ have been extracted from the CLEO data on the $\pi^{0} \gamma$ transition form factor used. For the high twist subprocess, we have taken $q_{1}+\bar{q}_{2} \rightarrow\left(q_{1} \bar{q}_{2}\right)+\gamma$. We have extracted the following four high twist subprocesses $u \bar{d} \rightarrow \pi^{+} \gamma, \bar{d} u \rightarrow \pi^{+} \gamma, \bar{u} d \rightarrow \pi^{-} \gamma$, $d \bar{u} \rightarrow \pi^{-} \gamma$, contributing to $p p \rightarrow \pi^{+}\left(\right.$or $\left.\pi^{-}\right) \gamma$ cross sections. As the dominant leading twist subprocess for the pion production, we have taken the quark-antiquark annihilation $q \bar{q} \rightarrow g \gamma$, where the $\pi$ meson is indirectly emitted from the gluon. The results of our numerical calculations have been plotted in Figs.3-18. As shown in Figs.3-4 and Figs.11-12, the high twist differential cross section monotonically decrease when the transverse momentum of the pion increases. As seen from Figs.3-4 and Figs.11-12 in all wave functions of mesons, the dependencies of the high twist cross sections on the $p_{T}$ transverse momentum of the pion demonstrate the same behavior. But, the higher twist corrections are very sensitive to the choice of the pion wave function. It should be noted that the magnitude of the high twist 
cross section for the pion wave function $\Phi_{C L E O}\left(x, Q^{2}\right)$ is very close to the asymptotic wave function $\Phi_{a s y}(x)$.

In Figs.5 and 13, the ratio $R=\Sigma_{\pi^{+}}^{H T} / \Sigma_{\pi^{+}}^{L T}$ has been plotted at $y=0$ as a function of the pion transverse momentum, $p_{T}$, for the different pion wave functions. It may be observed that the values of $R$ for fixed $y$ and $\sqrt{s}$ depend on the choice of pion wave function. Within this context, we have also calculated the distinction between $R\left(\Phi_{a s y}(x)\right)$ and $R\left(\Phi_{C L E O}\left(x, Q^{2}\right)\right), R\left(\Phi_{C Z}\left(x, Q^{2}\right)\right), R\left(\Phi_{P_{2}}\left(x, Q^{2}\right)\right)$ and $R\left(\Phi_{P_{3}}\left(x, Q^{2}\right)\right)$. We have ultimately found that the difference between $R\left(\Phi_{\text {asy }}(x)\right)$ and $R\left(\Phi_{C L E O}\left(x, Q^{2}\right)\right)$ is small, whereas a distinction between $R\left(\Phi_{a s y}(x)\right)$ with $R\left(\Phi_{C Z}\left(x, Q^{2}\right)\right), R\left(\Phi_{P_{2}}\left(x, Q^{2}\right)\right)$ and $R\left(\Phi_{P_{3}}\left(x, Q^{2}\right)\right)$ is significant. In Figs.6 and 14, we have shown the difference high twist cross section, $\Delta_{\pi}^{H T}=\Sigma_{\pi^{+}}^{H T}$ $\Sigma_{\pi^{-}}^{H T}$, as a function of the pion transverse momentum, $p_{T}$, for five different pion wave functions. As seen from Figs.6 and 14 when the transverse momentum of the pion increases, the difference of the high twist cross section, $\Delta_{\pi}^{H T}$, monotonically decreases. As shown in Figs.6 and $14, \Delta_{\pi}^{H T}$ in the $\Phi_{C L E O}\left(x, Q^{2}\right)$ pion wave function is very close to that of the asymptotic wave function $\Phi_{a s y}(x)$.

In Figs.8 and 16, the ratio $r=\Delta_{\pi}^{H T} / \Delta_{\pi}^{L T}$ is plotted at $y=0$ as a function of the pion transverse momentum, $p_{T}$, for five pion wave functions. As shown in Figs.8 and 16, the values of $r$ for fixed $y$ and $\sqrt{s}$ depend on the choice of the pion wave function similar to Fig.5 and 13. We have also calculated the distinction between $r\left(\Phi_{a s y}(x)\right)$ and $r\left(\Phi_{C L E O}\left(x, Q^{2}\right)\right)$, $r\left(\Phi_{C Z}\left(x, Q^{2}\right)\right), r\left(\Phi_{P_{2}}\left(x, Q^{2}\right)\right)$ and $r\left(\Phi_{P_{3}}\left(x, Q^{2}\right)\right)$. For all transverse momentum $p_{T}$ of the pion in the center-of-mass energy $\sqrt{s}=63 \mathrm{GeV}$ and also in $630 \mathrm{GeV}$, we have obtained the following interesting relation:

$$
\frac{R\left(\Phi_{i}\left(x, Q^{2}\right)\right)}{R\left(\Phi_{a s y}(x)\right)}=\frac{r\left(\Phi_{i}\left(x, Q^{2}\right)\right)}{r\left(\Phi_{\text {asy }}(x)\right)}, \quad\left(i=C L E O, C Z, P_{2} . P_{3}\right)
$$

In Figs.9-10 and Figs.17-18, we have depicted $\Sigma_{\pi^{+}}^{H T}$ high twist and difference high twist cross sections $\Delta_{\pi}^{H T}=\Sigma_{\pi^{+}}^{H T} \Sigma_{\pi^{-}}^{H T}$ as a function of the rapidity $y$ of the pion at $\sqrt{s}=63 \mathrm{GeV}, 630 \mathrm{GeV}$ and $p_{T}=5 \mathrm{GeV} / \mathrm{c}, 50 \mathrm{GeV} / \mathrm{c}$, respectively. As we are now in the high energy region, the change of the rapidity of this relation may be expressed as folows: $-\ln \left(\sqrt{s} / p_{T}\right) \leq y \leq$ $\ln \left(\sqrt{s} / p_{T}\right)$. At $\sqrt{s}=63 \mathrm{GeV}, p_{T}=5 \mathrm{GeV} / \mathrm{c}$ and $\sqrt{s}=630 \mathrm{GeV}, p_{T}=50 \mathrm{GeV} / \mathrm{c}$ the pion rapidity lies in the region $-2.52 \leq y \leq 2.52$. As seen from Figs.9-10 and Figs.17-18 in the region $(-2.52 \leq y \leq 0.85)$, the high twist cross section for the asymptotic $\Phi_{\text {asy }}(x)$ and $\Phi_{C L E O}\left(x, Q^{2}\right)$ wave functions increases and it has a maximum approximately at one point 
$y=0.85$. After that, the cross sections monotonically decrease with an increase in the $y$ rapidity of the pion. As seen from Figs.9-10 and Figs.17-18, the $\Sigma_{\pi^{+}}^{H T}$ and the $\Delta_{\pi}^{H T}$ cross sections are very sensitive to the choice of the meson wave functions. Also, as shown in Figs.9, 10, 17 and 18 in the regions $-2.52 \leq y \leq-1$ and $1 \leq y \leq 2.52$ the high twist cross section for the $\Phi_{C L E O}\left(x, Q^{2}\right)$ is very close to the asymptotic wave function $\Phi_{a s y}(x)$ case. Our investigation enables us to conclude that the high twist pion production cross section in the

proton-proton collisions depends on the form of the pion model wave functions and may be used for their study. Further investigations are needed in order to clarify the role of high twist effects in QCD.

\section{Acknowledgments}

Two of authors, A. I. Ahmadov and I. Boztosun are grateful to TÜBİTAK Grant-2221 (BAYG) as well as TÜBİTAK Grant: TBAG-2398. One of us, A. I. Ahmadov is also grateful to NATO Reintegration Grant-980779

\section{REFERENCES}

[1] A.V. Radyushkin, Dubna preprint P2-10717, 1977, hep-ph/0410276.

[2] V. L. Chernyak and A. R. Zhitnitsky, Nucl. Phys. B201, 492 (1982); V. L. Chernyak, A. R. Zhitnitsky and I. R. Zhitnitsky, Nucl. Phys. B204, 477 (1982).

[3] V. L. Chernyak and A. R. Zhitnitsky, Nucl. Phys. B246, 52 (1984).

[4] I. D. King, C. T. Sachrajda, Nucl. Phys. B279, 785 (1987).

[5] V. L. Chernyak, A. R. Zhitnitsky, Phys. Rept. 112, 173 (1984).

[6] S. V. Mikhailov and A. V. Radyushkin, JETP Lett. 43, 712 (1986); Sov. J. Nucl. Phys.49, 494 (1989); Phys. Rev. D45, 1754 (1992).

[7] V. M. Braun and I. E. Filyanov, Z. Phys. C44, 157 (1989).

[8] G. R. Farrar, K. Huleihel and H. Zhang, Nucl. Phys. B349, 655 (1991).

[9] V. L. Chernyak, A. A. Ogloblin and I. R. Zhitnitsky, Z. Phys. C42, 583 (1989).

[10] G. R. Farrar, H. Zhang, A. A. Ogloblin and I. R. Zhitnitsky, Nucl. Phys. B311, 585 (1988).

[11] M. Anselmino, P. Kroll and B. Pire, Z. Phys. C36, 89 (1987). 
[12] A. V. Radyushkin and R. Ruskov, Phys. Lett. B374, 173 (1996); Nucl. Phys. B481, 625 (1996).

[13] The CLEO Collaboration (J.Gronberg et.al.), Phys. Rev. D57, 33 (1998).

[14] V. Yu. Petrov, M. V. Polyakov, R. Ruskov, C. Weiss and K. Goeke, Phys. Rev. D59, 114018 (1999); hep-ph/9807229.

[15] J. Qiu and G. Sterman, Nucl. Phys. B353, 105, 137 (1991).

[16] E. L. Berger and S. J. Brodsky, Phys. Rev. Lett. 42, 940 (1979); E. L. Berger, Z. Phys. C4, 289 (1980).

[17] R. Doria, J. Frenkel and J. C. Taylor, Nucl. Phys. B168, 93 (1980); F. T. Brandt, J. Frenkel and J. C. Taylor, Nucl. Phys. B312, 589 (1989).

[18] K. Kastella, G. Sterman and J. Milana, Phys. Rev. D39, 2586 (1989).

[19] G. P. Lepage and S. J. Brodsky, Phys. Rev. D22, 2157 (1980).

[20] A. Schmedding and O. Yakovlev, Phys. Rev. D62, 116002 (2000), hep-ph/9905392.

[21] V. L. Chernyak, Preprint TPI-MINN-91-47-T, December 1991.

[22] J. F. Owens, Rev. Mod. Phys. 59, 465 (1987).

[23] R. Kh. Muradov and A. I. Ahmadov, Central. Eur. J. Phys. 3(3) (2005) 433, hep-ph/0412227.

[24] A. I. Ahmadov, I. Boztosun, R. Kh. Muradov, A. Soylu and E. A. Dadashov, hep-ph/0604172.

[25] MRST2003c.f can be obtained from http://durpdg.dur.ac.uk/hepdata/pdf.html. See also, A. D. Martin, R. G. Roberts, W. J. Stirling and R. S.Thorne, hep-ph/0307262; R. S.Thorne, hep-ph/0309343.

[26] B. A. Kniehl, G. Kramer, B. Pötter, Nucl.Phys. B582, 514 (2000), hep-ph/0010289. 


\begin{tabular}{|c|c|c|c|c|}
\hline$p_{T}, G e V / c$ & $\frac{R\left(\Phi_{C L E O}\left(x, Q^{2}\right)\right)}{R\left(\Phi_{a s y}(x)\right)}$ & $\frac{R\left(\Phi_{C Z}\left(x, Q^{2}\right)\right)}{R\left(\Phi_{a s y}(x)\right)}$ & $\frac{R\left(\Phi_{P_{2}}\left(x, Q^{2}\right)\right)}{R\left(\Phi_{a s y}(x)\right)}$ & $\frac{R\left(\Phi_{P_{3}}\left(x, Q^{2}\right)\right)}{R\left(\Phi_{a s y}(x)\right)}$ \\
\hline 2 & 1.496 & 8.435 & 10.569 & 16.525 \\
\hline 6 & 3.358 & 3.888 & 4.617 & 3.001 \\
\hline 20 & 0.9476 & 0.382987 & 0.302073 & 0.133824 \\
\hline
\end{tabular}

TABLE I: The distinction between $R\left(\Phi_{a s y}(x)\right)$ with $R\left(\Phi_{i}\left(x, Q^{2}\right)\right)$ (i=CLEO, CZ, $\left.P_{2}, P_{3}\right)$ at c.m. energy $\sqrt{s}=63 \mathrm{GeV}$.

\begin{tabular}{|c|c|c|c|c|}
\hline$p_{T}, G e V / c$ & $\frac{r\left(\Phi_{C L E O}\left(x, Q^{2}\right)\right)}{r\left(\Phi_{a s y}(x)\right)}$ & $\frac{r\left(\Phi_{C Z}\left(x, Q^{2}\right)\right)}{r\left(\Phi_{a s y}(x)\right)}$ & $\frac{r\left(\Phi_{P_{2}}\left(x, Q^{2}\right)\right)}{r\left(\Phi_{a s y}(x)\right)}$ & $\frac{r\left(\Phi_{P_{3}}\left(x, Q^{2}\right)\right)}{r\left(\Phi_{a s y}(x)\right)}$ \\
\hline 2 & 1.496 & 8.435 & 10.568 & 16.524 \\
\hline 6 & 3.358 & 3.887 & 4.616 & 3.001 \\
\hline 20 & 0.95 & 0.38315 & 0.302109 & 0.13384 \\
\hline
\end{tabular}

TABLE II: The distinction between $r\left(\Phi_{a s y}(x)\right)$ with $r\left(\Phi_{i}\left(x, Q^{2}\right)\right)$ (i=CLEO, CZ, $\left.P_{2}, P_{3}\right)$ at c.m. energy $\sqrt{s}=63 \mathrm{GeV}$.

\begin{tabular}{|c|c|c|c|c|}
\hline$p_{T}, G e V / c$ & $\frac{R\left(\Phi_{C L E O}\left(x, Q^{2}\right)\right)}{R\left(\Phi_{a s y}(x)\right)}$ & $\frac{R\left(\Phi_{C Z}\left(x, Q^{2}\right)\right)}{R\left(\Phi_{a s y}(x)\right)}$ & $\frac{R\left(\Phi_{P_{2}}\left(x, Q^{2}\right)\right)}{R\left(\Phi_{a s y}(x)\right)}$ & $\frac{R\left(\Phi_{P_{3}}\left(x, Q^{2}\right)\right)}{R\left(\Phi_{a s y}(x)\right)}$ \\
\hline 20 & 1.531 & 5.582 & 6.815 & 9.63 \\
\hline 60 & 2.202 & 2.591 & 2.961 & 2.262 \\
\hline 200 & 0.926 & 0.605 & 0.544 & 0.43 \\
\hline
\end{tabular}

TABLE III: The distinction between $R\left(\Phi_{\text {asy }}(x)\right)$ with $R\left(\Phi_{i}\left(x, Q^{2}\right)\right)$ (i=CLEO, CZ, $\left.P_{2}, P_{3}\right)$ at c.m. energy $\sqrt{s}=630 \mathrm{GeV}$. 


\begin{tabular}{|c|c|c|c|c|}
\hline$p_{T}, G e V / c$ & $\frac{r\left(\Phi_{C L E O}\left(x, Q^{2}\right)\right)}{r\left(\Phi_{a s y}(x)\right)}$ & $\frac{r\left(\Phi_{C Z}\left(x, Q^{2}\right)\right)}{r\left(\Phi_{a s y}(x)\right)}$ & $\frac{r\left(\Phi_{P_{2}}\left(x, Q^{2}\right)\right)}{r\left(\Phi_{a s y}(x)\right)}$ & $\frac{r\left(\Phi_{P_{3}}\left(x, Q^{2}\right)\right)}{r\left(\Phi_{a s y}(x)\right)}$ \\
\hline 20 & 1.531 & 5.583 & 6.817 & 9.632 \\
\hline 60 & 2.202 & 2.591 & 2.961 & 2.262 \\
\hline 200 & 0.926 & 0.605 & 0.544 & 0.43 \\
\hline
\end{tabular}

TABLE IV: The distinction between $r\left(\Phi_{a s y}(x)\right)$ with $\left.r\left(\Phi_{i}\left(x, Q^{2}\right)\right)\left(\mathrm{i}=\mathrm{CLEO}, \mathrm{CZ}, P_{2}, P_{3}\right)\right)$ at c.m. energy $\sqrt{s}=630 \mathrm{GeV}$. 


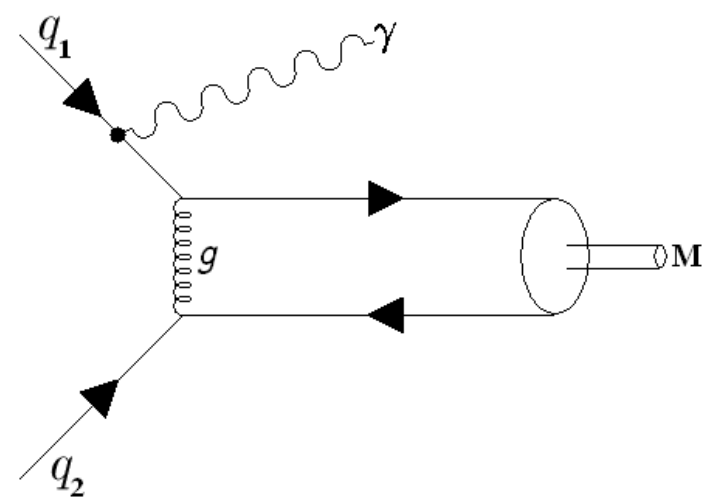

(a)

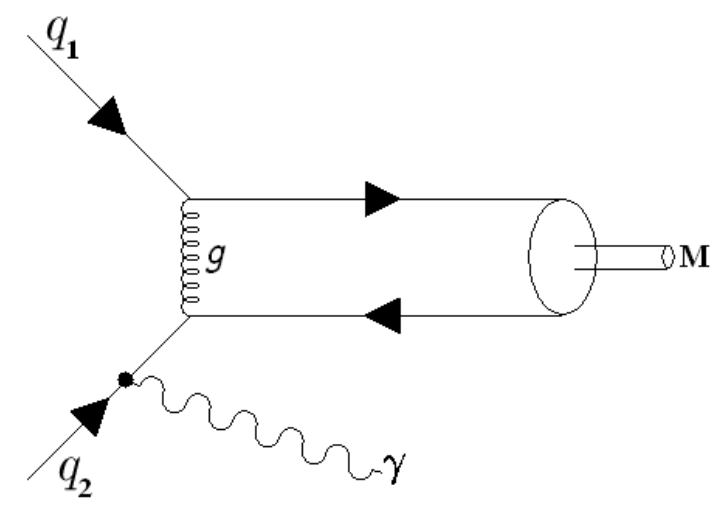

(c)

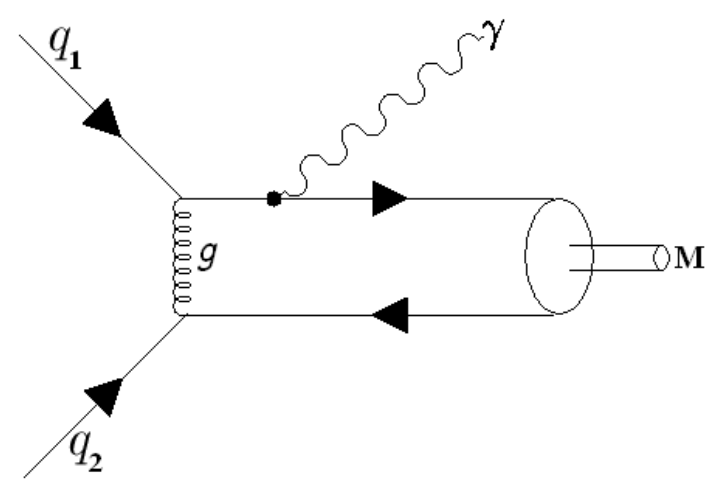

(b)

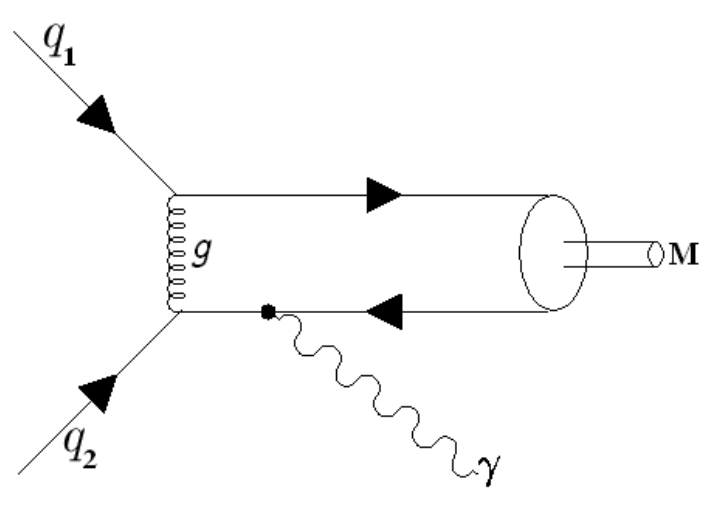

(d)

FIG. 1: Feynman diagrams for the high twist subprocess, $q_{1} q_{2} \rightarrow \pi^{+}\left(\right.$or $\left.\pi^{-}\right) \gamma$

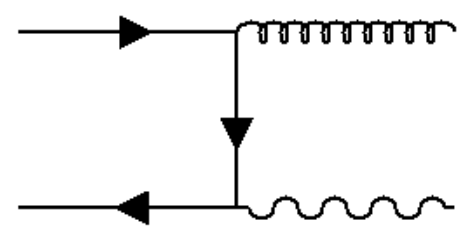

(a)

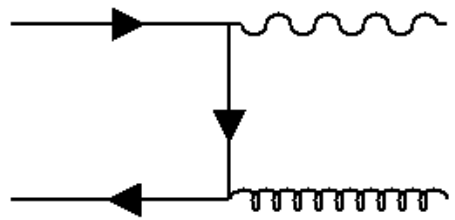

(b)

FIG. 2: Feynman diagrams for the leading twist subprocess, $q \bar{q} \rightarrow g \gamma, g \rightarrow M$. 


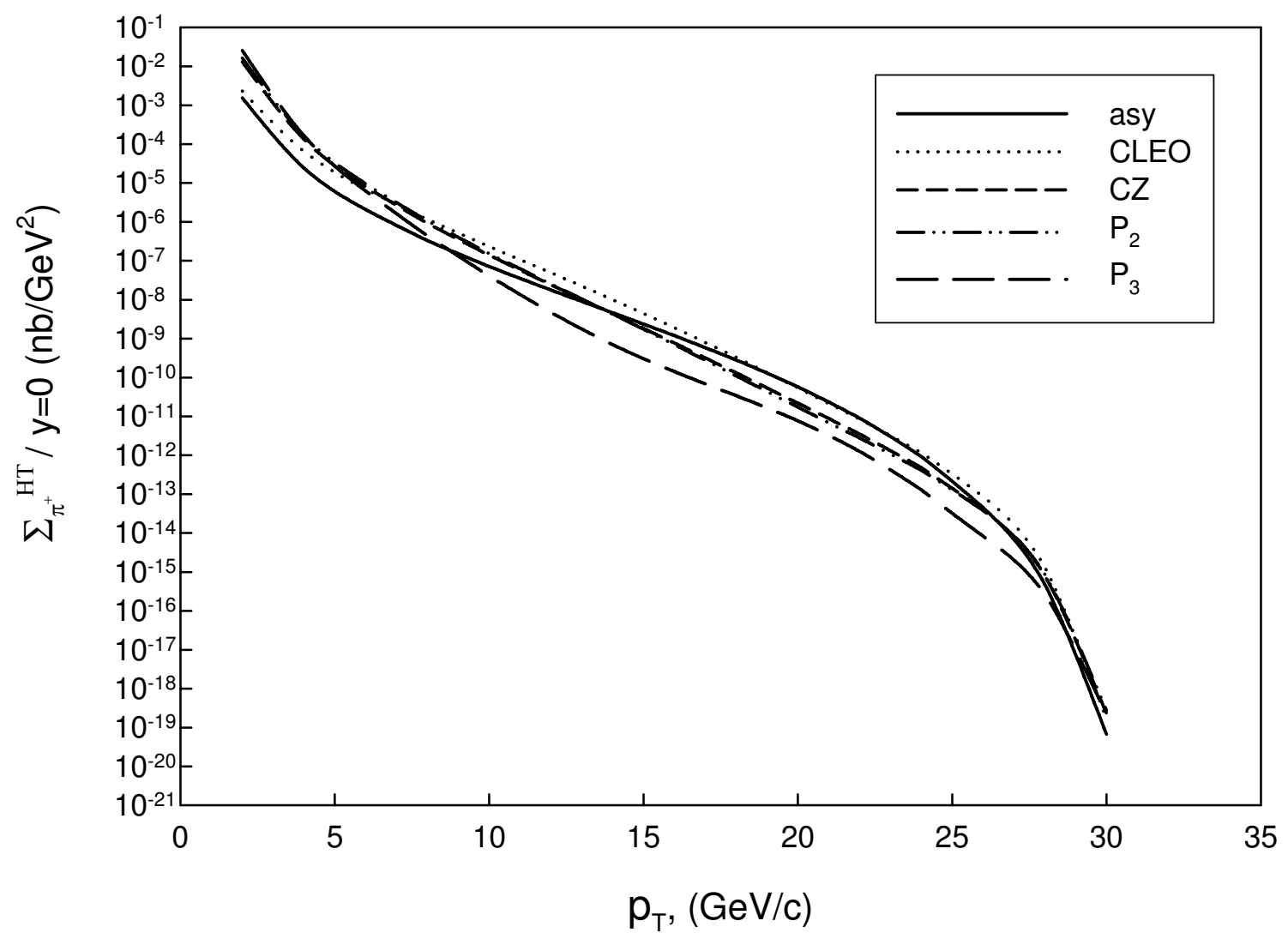

FIC 2: Hioh twist $\pi^{+}$nroduction cross sections as a function of the $n_{T}$ transverse momentum of

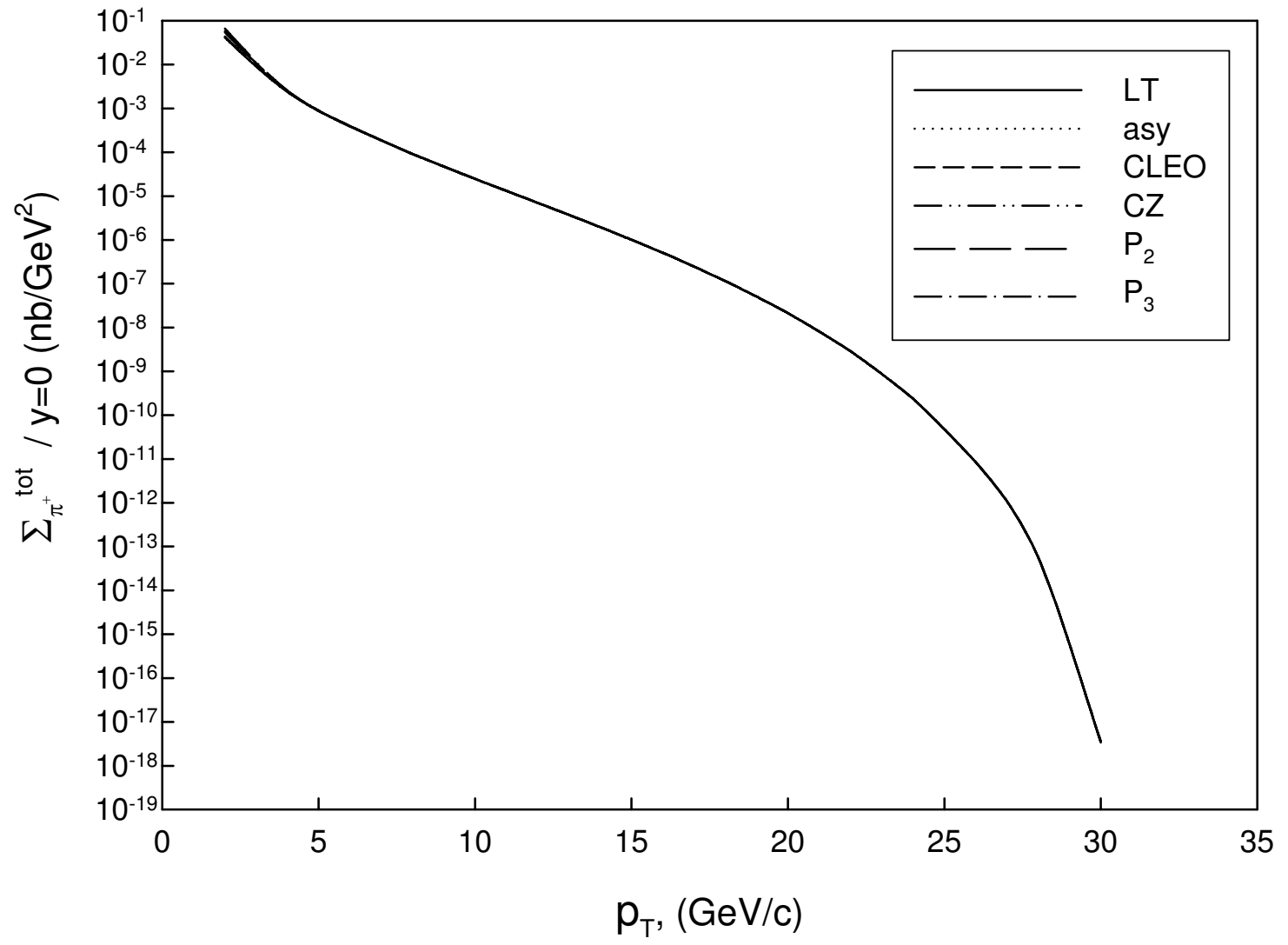

FIG. 4: The sum of the leading and the high twist $\pi^{+}$production cross sections $\Sigma_{\pi^{+}}^{t o t}=\Sigma_{\pi^{+}}^{L T}+\Sigma_{\pi^{+}}^{H T}$ as a function of the $p_{T}$ transverse momentum of the pion, at the c.m. energy $\sqrt{s}=63 G e V$. 


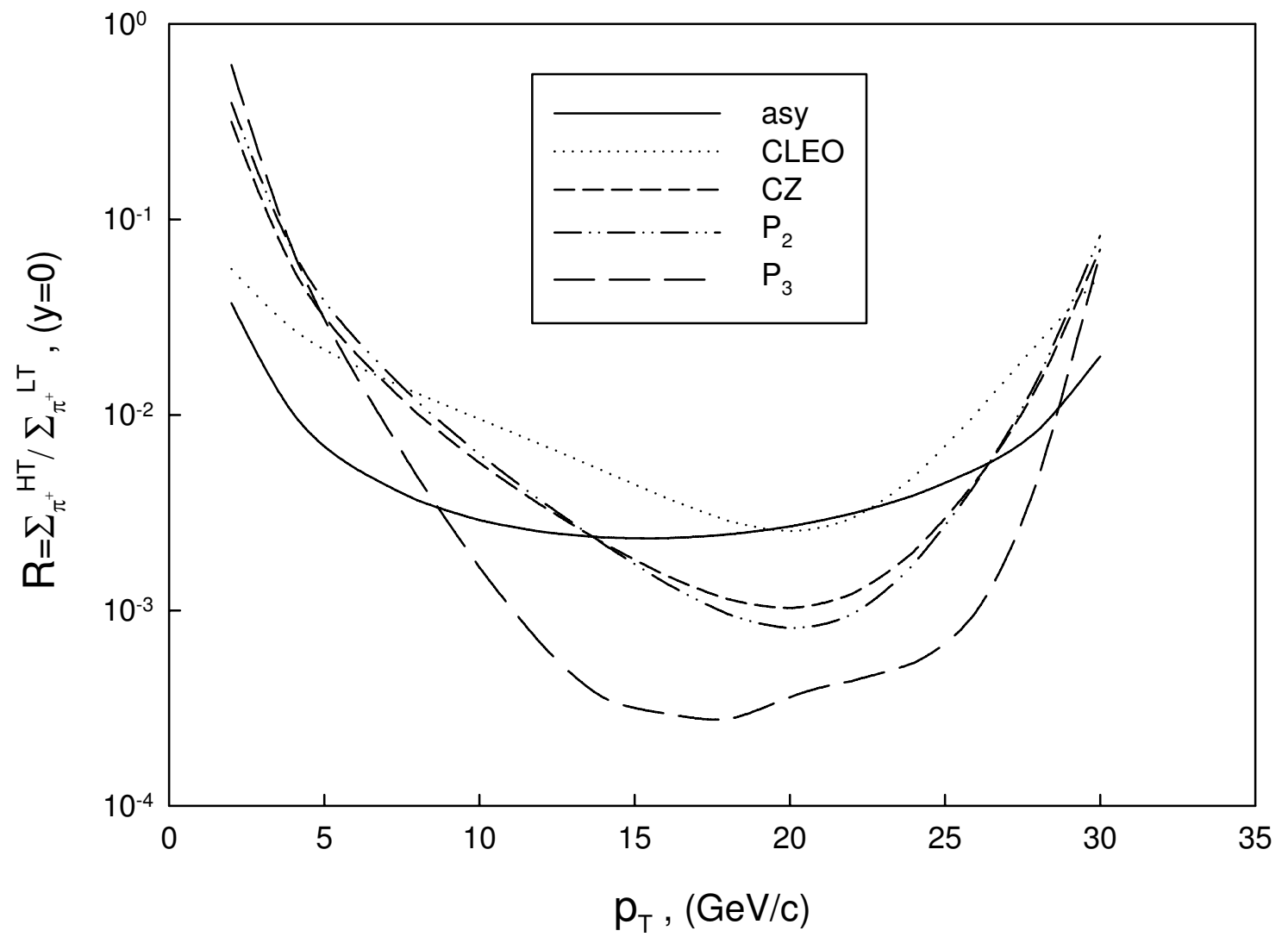

FIG. 5: Ratio $R=\Sigma_{\pi^{+}}^{H T} / \Sigma_{\pi^{+}}^{L T}$, where the leading and the high twist contributions are calculated for the pion rapidity $y=0$ at the c.m. energy $\sqrt{s}=63 \mathrm{GeV}$, as a function of the pion transverse momentum, $p_{T}$.

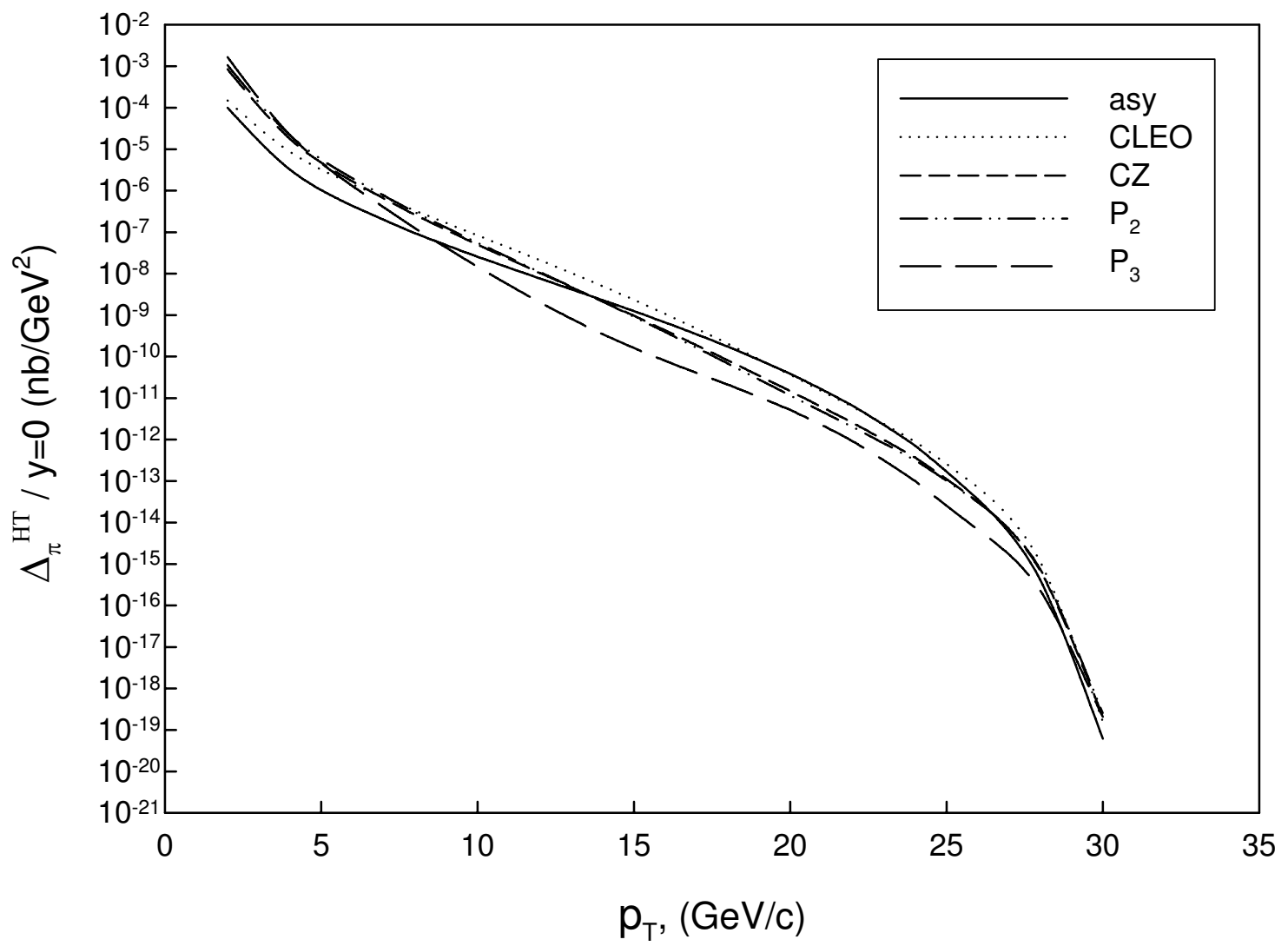

FIG. 6: The difference of the high twist cross section, $\Delta_{\pi}^{H T}=\Sigma_{\pi^{+}}^{H T}-\Sigma_{\pi^{-}}^{H T}$, as a function of the pion transverse momentum, $p_{T}$, at the c.m. energy $\sqrt{s}=63 \mathrm{GeV}$. 


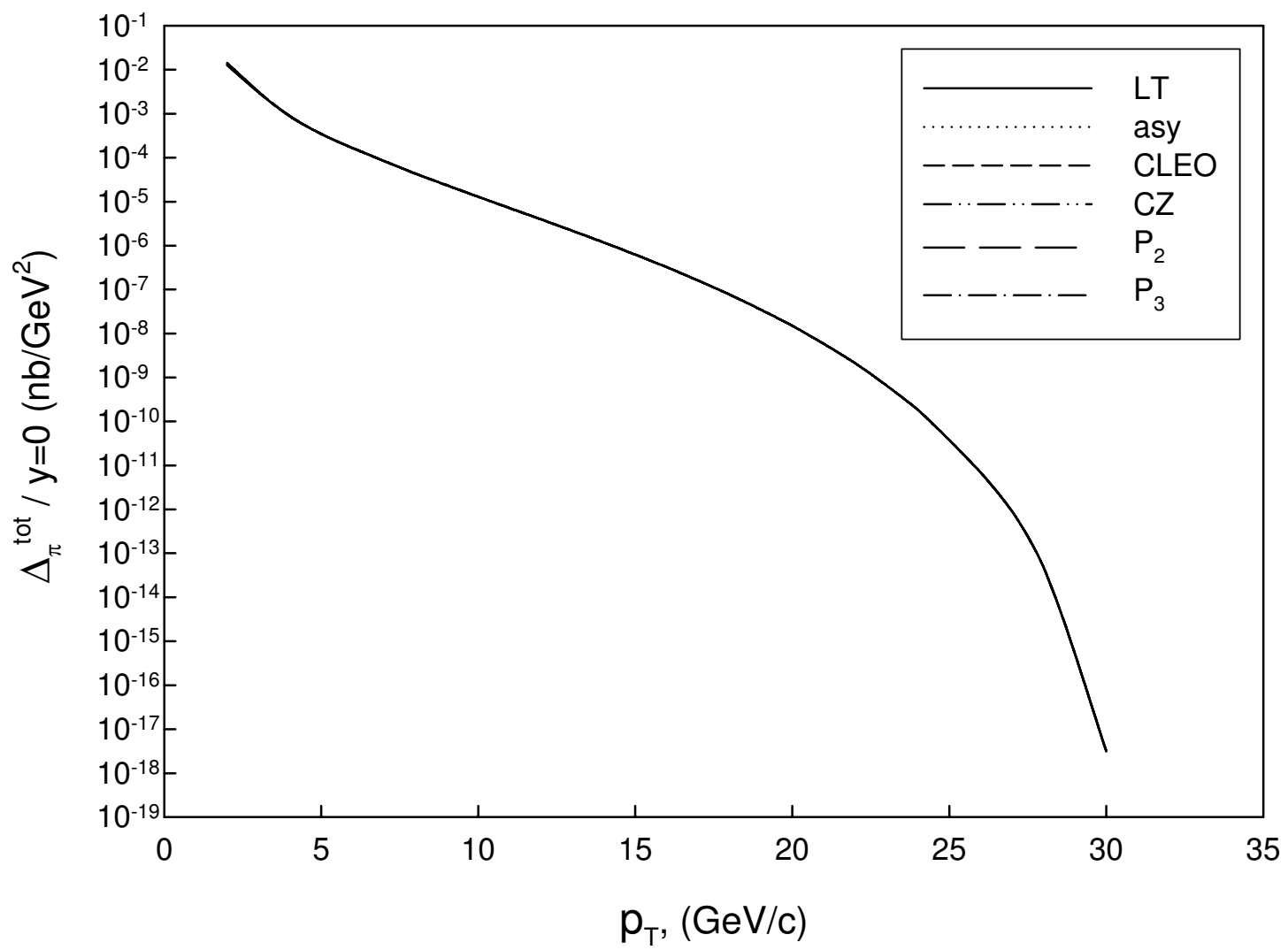

FIG. 7: The sum of the difference leading and high twist cross sections $\Delta_{\pi}^{t o t}=\Delta_{\pi}^{L T}+\Delta_{\pi}^{H T}$ as a function of the pion transverse momentum $p_{T}$ at the c.m. energy $\sqrt{s}=63 \mathrm{GeV}$.

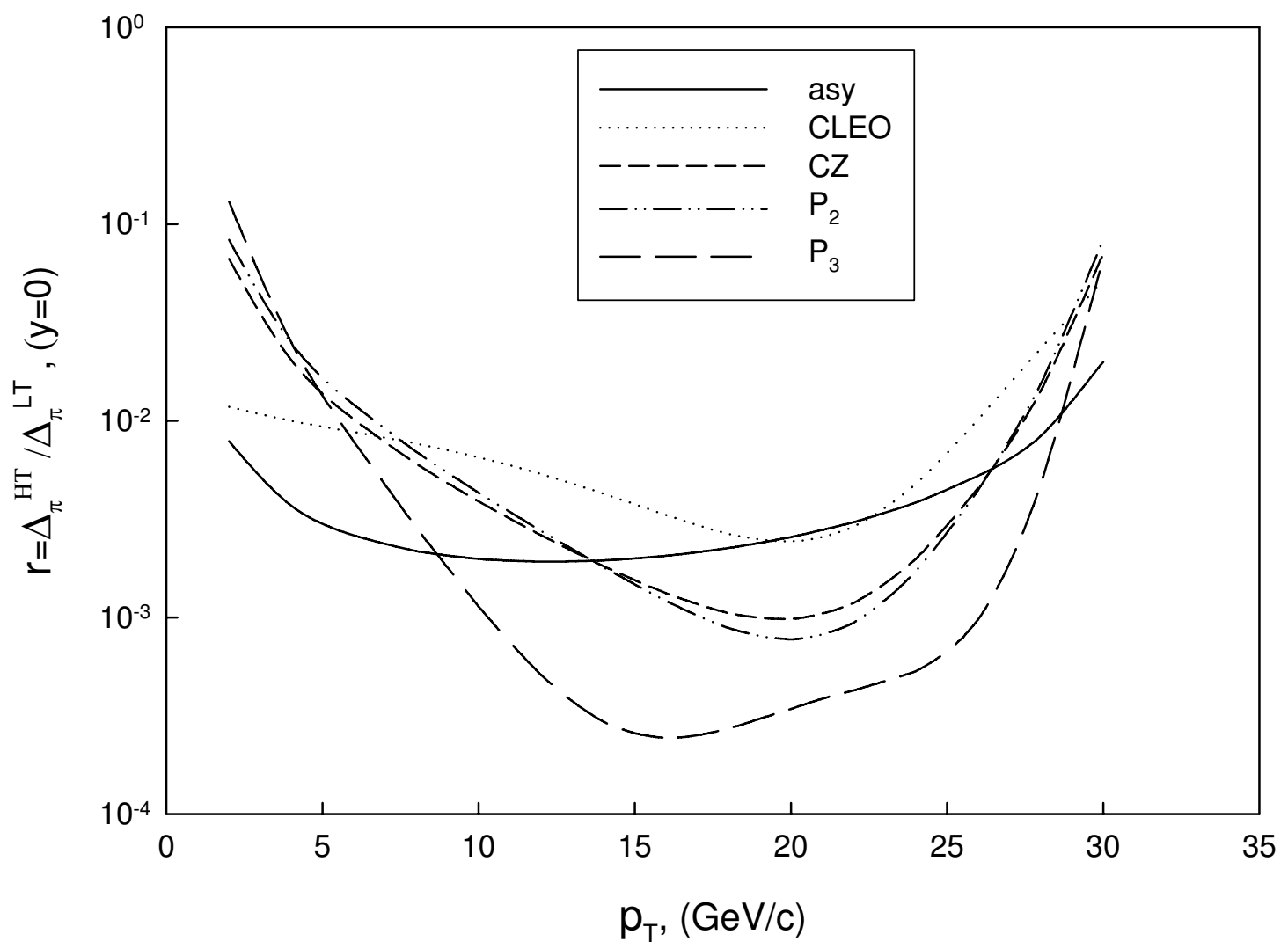

FIG. 8: The ratio $r=\Delta_{\pi}^{H T} / \Delta_{\pi}^{L T}$, where the leading and the high twist contributions are calculated for the pion rapidity $y=0$, at the c.m. energy $\sqrt{s}=63 G e V$, as a function of the pion transverse momentum $p_{T}$. 


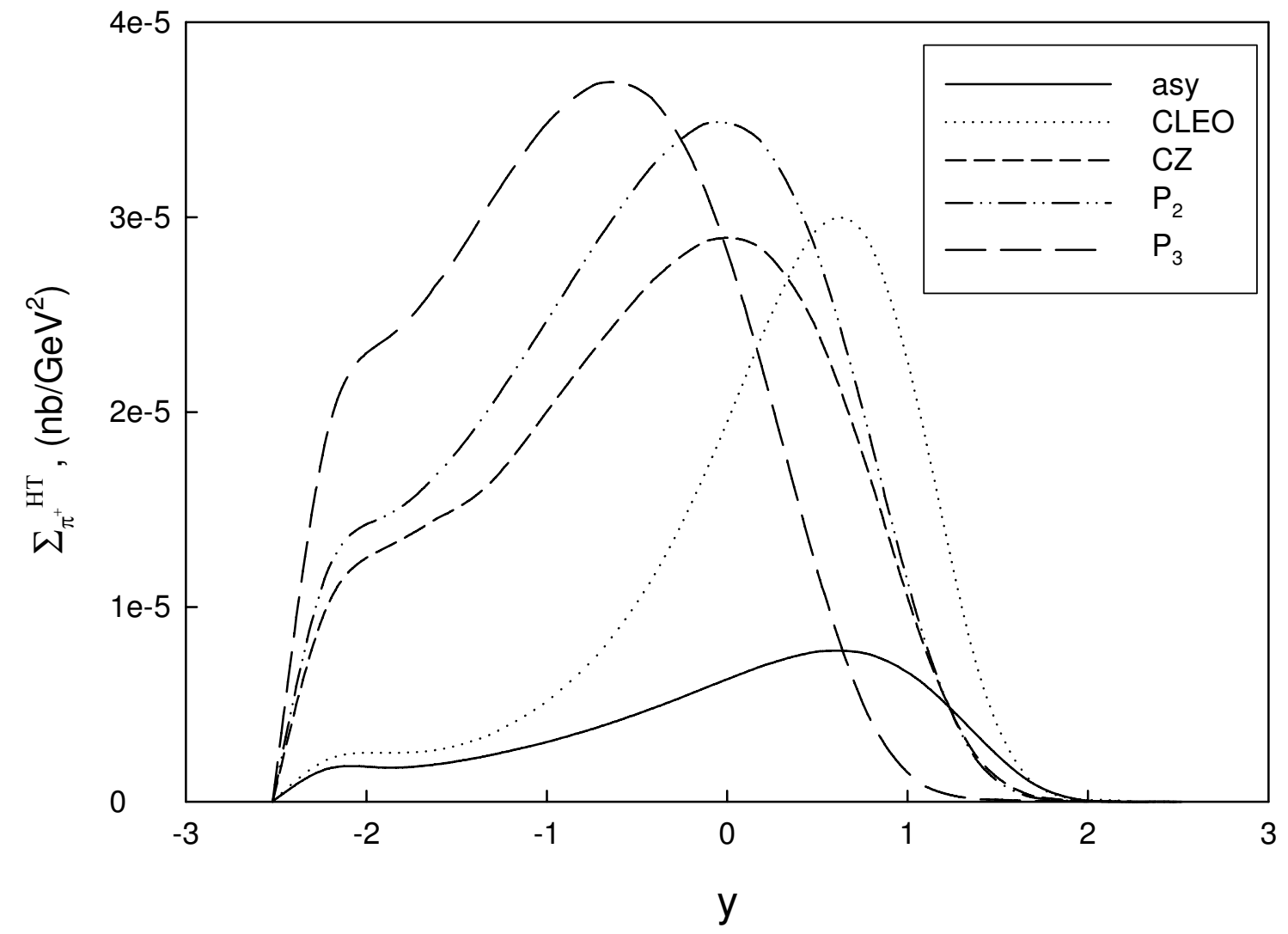

FIG. 9: High twist $\pi^{+}$production cross sections as a function of the $y$ rapidity of the pion at the transverse momentum of the pion $p_{T}=5 \mathrm{GeV}$, at the c.m.energy $\sqrt{s}=63 \mathrm{GeV}$.

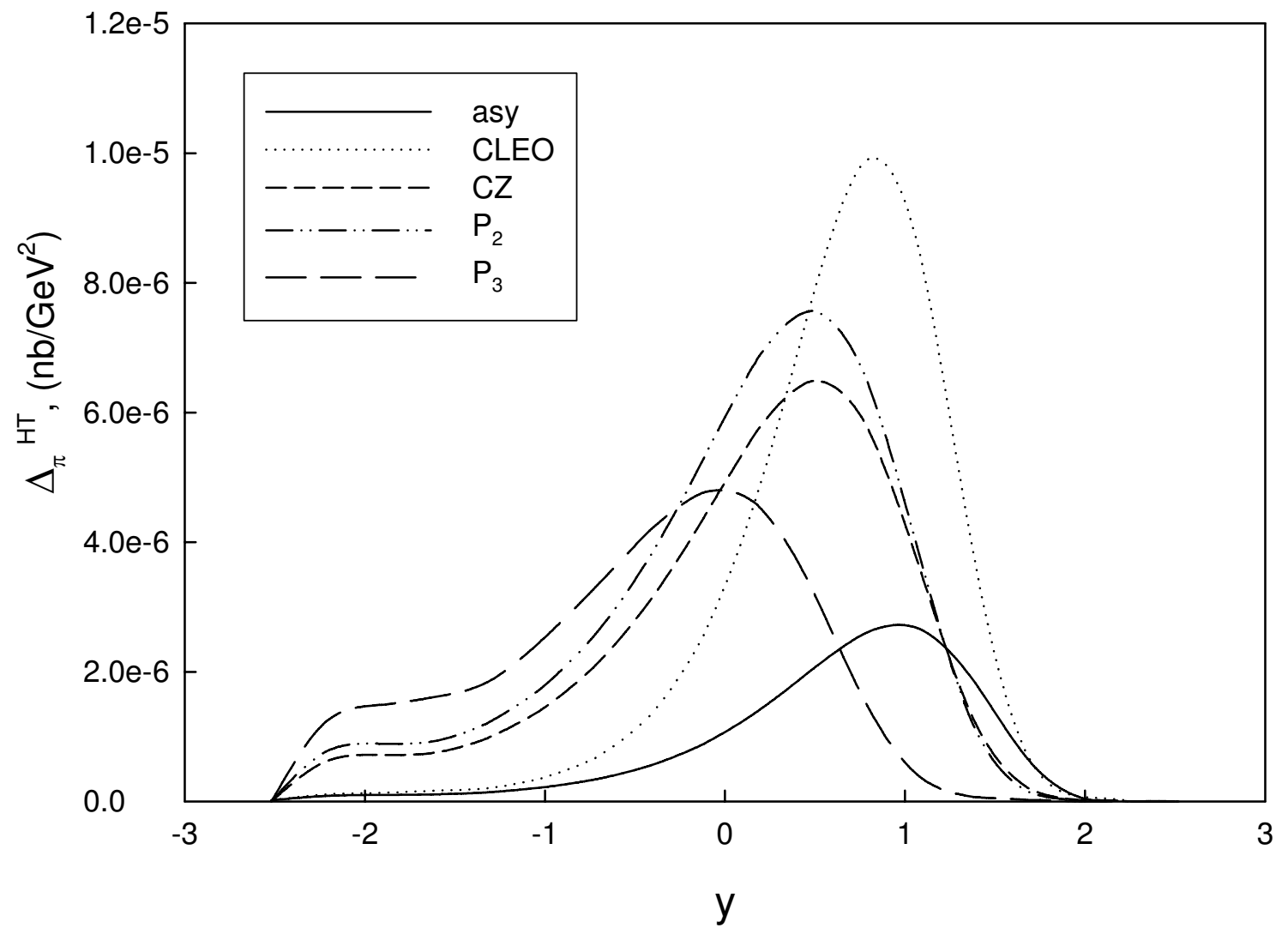

FIG. 10: The difference of the high twist cross section, $\Delta_{\pi}^{H T}=\Sigma_{\pi^{+}}^{H T}-\Sigma_{\pi^{-}}^{H T}$, as a function of the $y$ rapidity of the pion at the transverse momentum of the pion $p_{T}=5 G e V$, at the c.m. energy $\sqrt{s}=63 G e V$. 


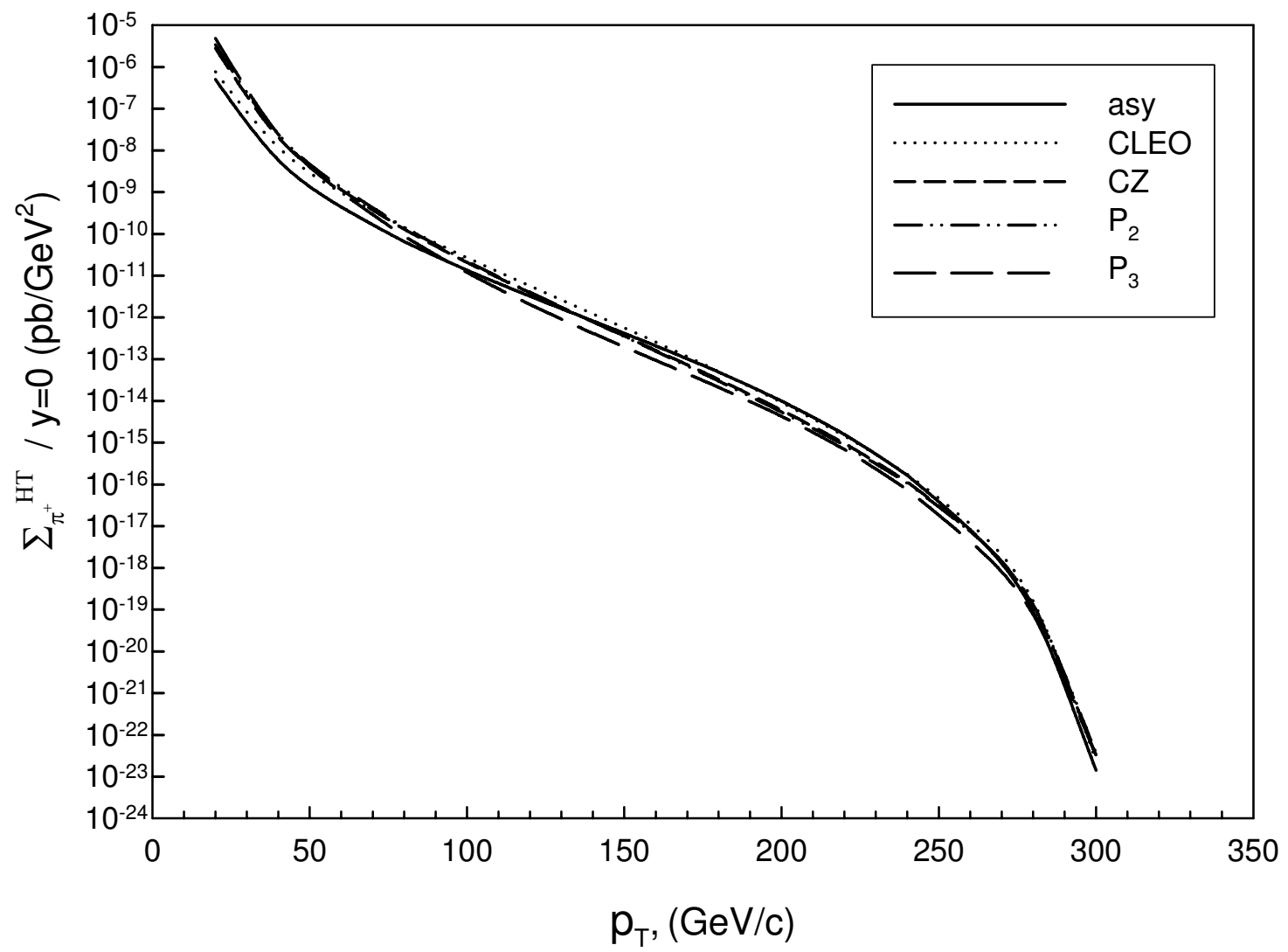

FIG. 11: High twist $\pi^{+}$production cross sections as a function of the $p_{T}$ transverse momentum of the pion at the c.m. energy $\sqrt{s}=630 \mathrm{GeV}$.

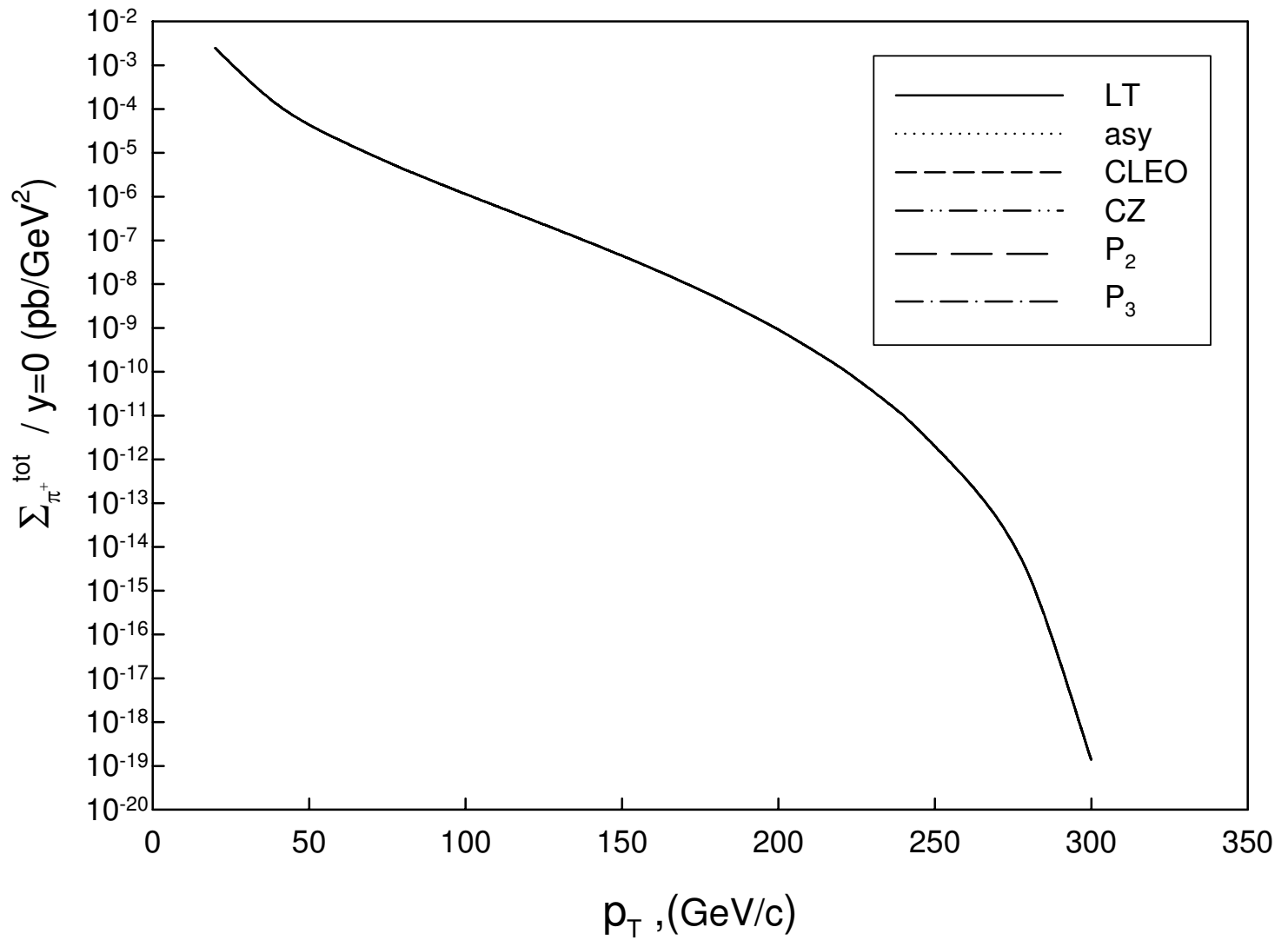

FIG. 12: The sum of the leading and the high twist $\pi^{+}$production cross sections $\Sigma_{\pi^{+}}^{t o t}=\Sigma_{\pi^{+}}^{L T}+\Sigma_{\pi^{+}}^{H T}$ as a function of the $p_{T}$ transverse momentum of the pion, at the c.m. energy $\sqrt{s}=630 \mathrm{GeV}$. 


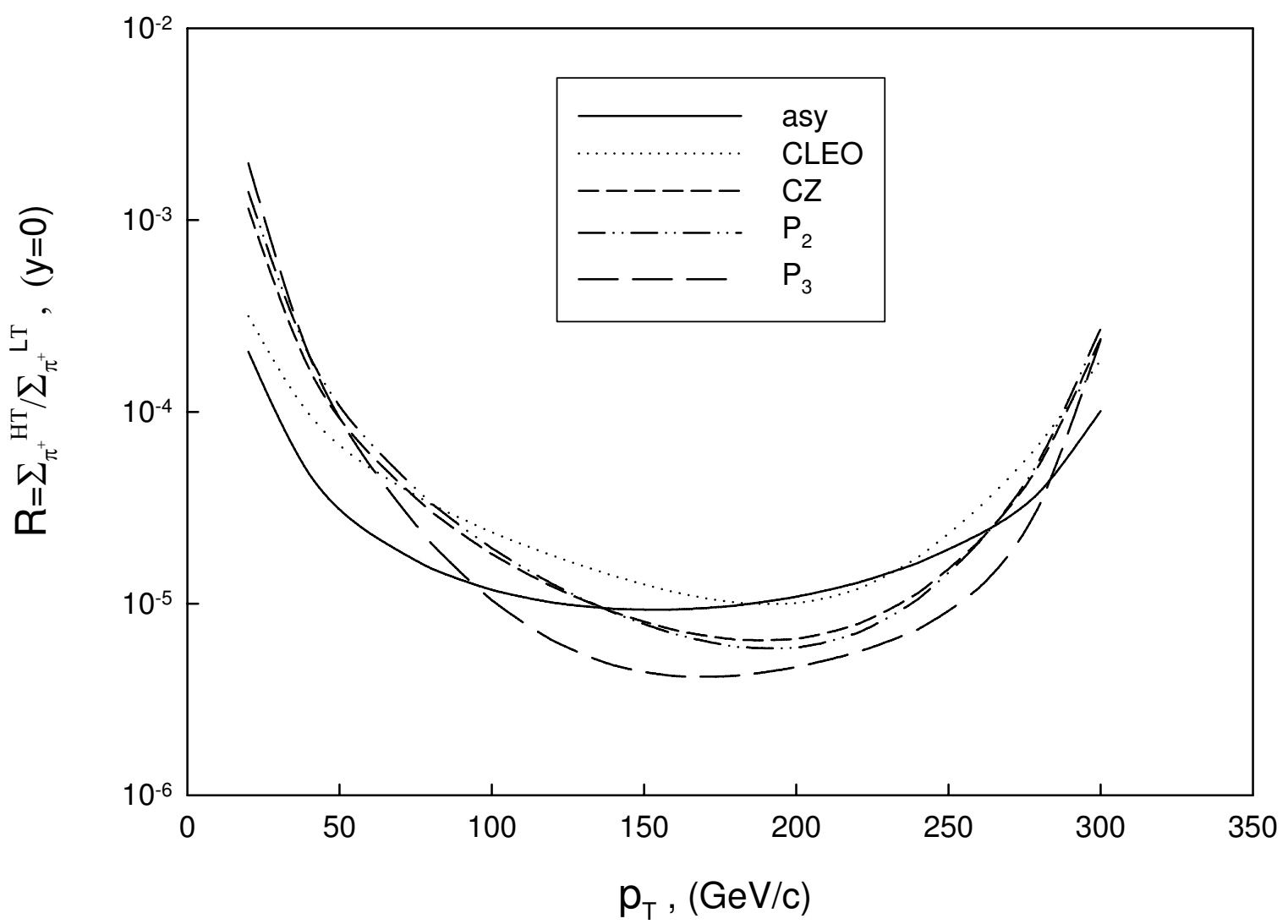

FIG. 13: The ratio $R=\Sigma_{\pi^{+}}^{H T} / \Sigma_{\pi^{+}}^{L T}$, where the leading and the high twist contributions are calculated for the pion rapidity $y=0$ at the c.m. energy $\sqrt{s}=630 \mathrm{GeV}$ as a function of the pion transverse momentum $p_{T}$.

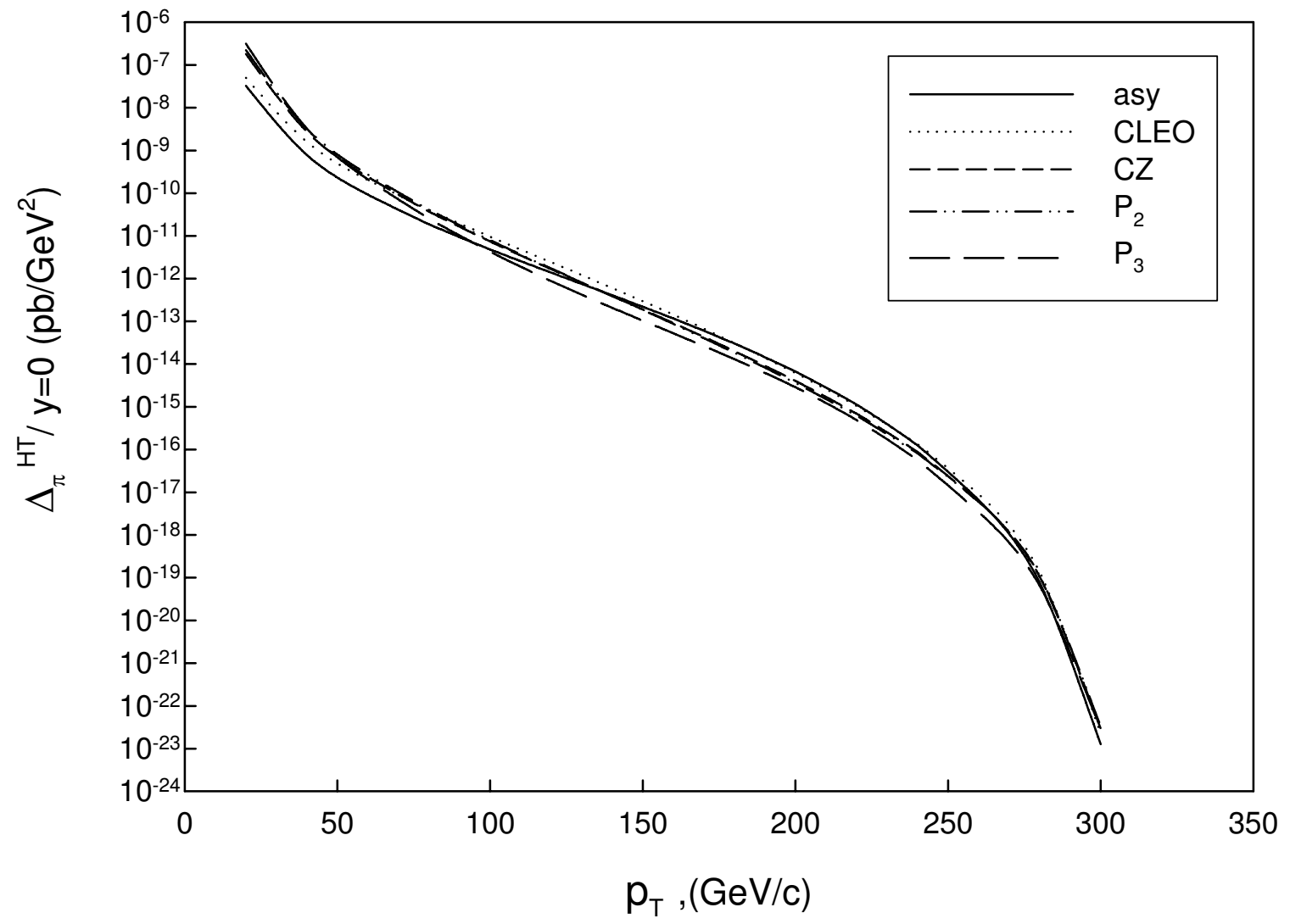

FIG. 14: The difference of the high twist cross section, $\Delta_{\pi}^{H T}=\Sigma_{\pi^{+}}^{H T}-\Sigma_{\pi^{-}}^{H T}$, as a function of the pion transverse momentum, $p_{T}$, at the c.m. energy $\sqrt{s}=630 \mathrm{GeV}$. 


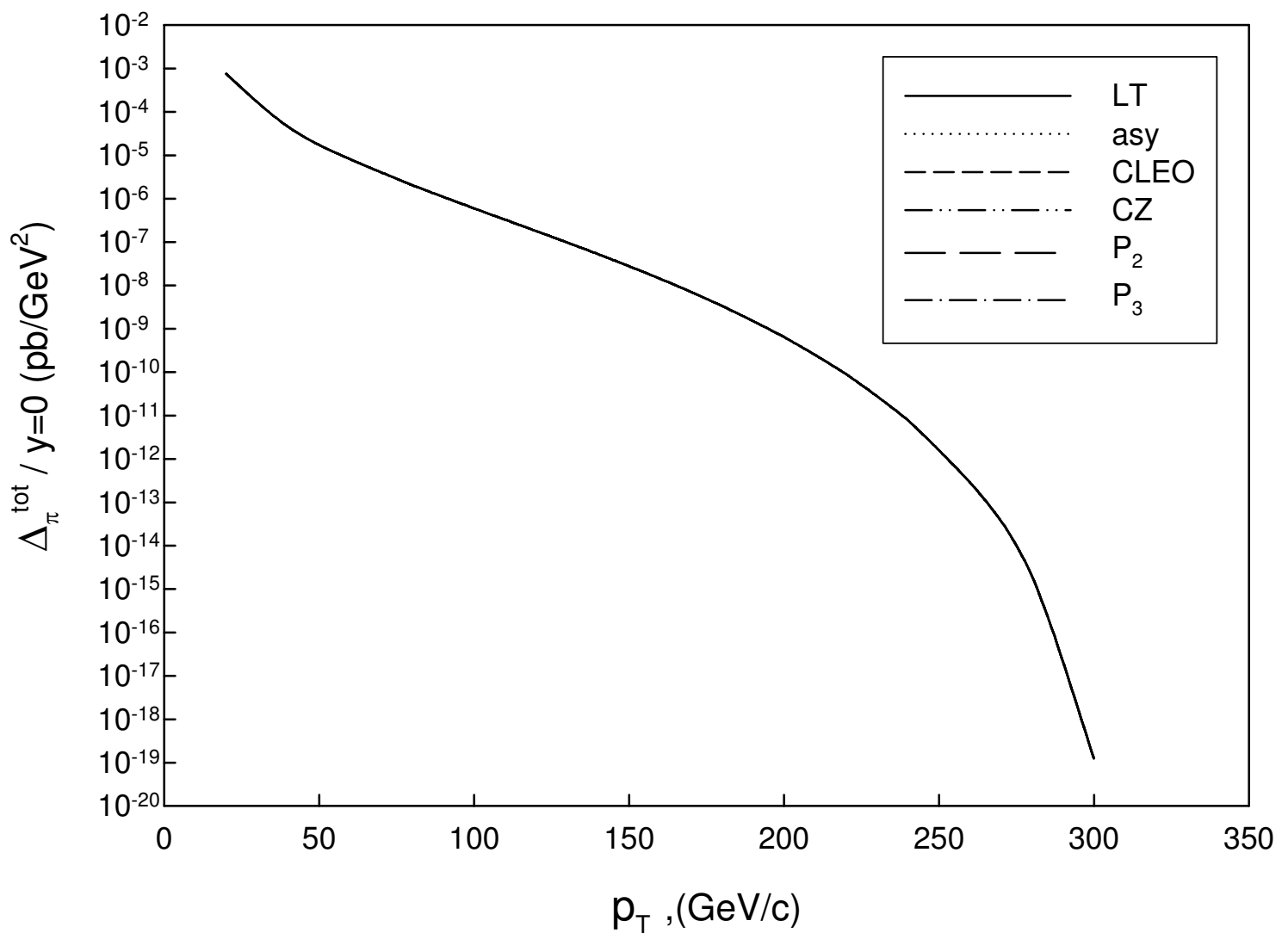

FIG. 15: The sum of the difference of the leading and the high twist cross sections, $\Delta_{\pi}^{t o t}=\Delta_{\pi}^{L T}+\Delta_{\pi}^{H T}$, as a function of the pion transverse momentum, $p_{T}$, at the c.m. energy $\sqrt{s}=630 \mathrm{GeV}$.

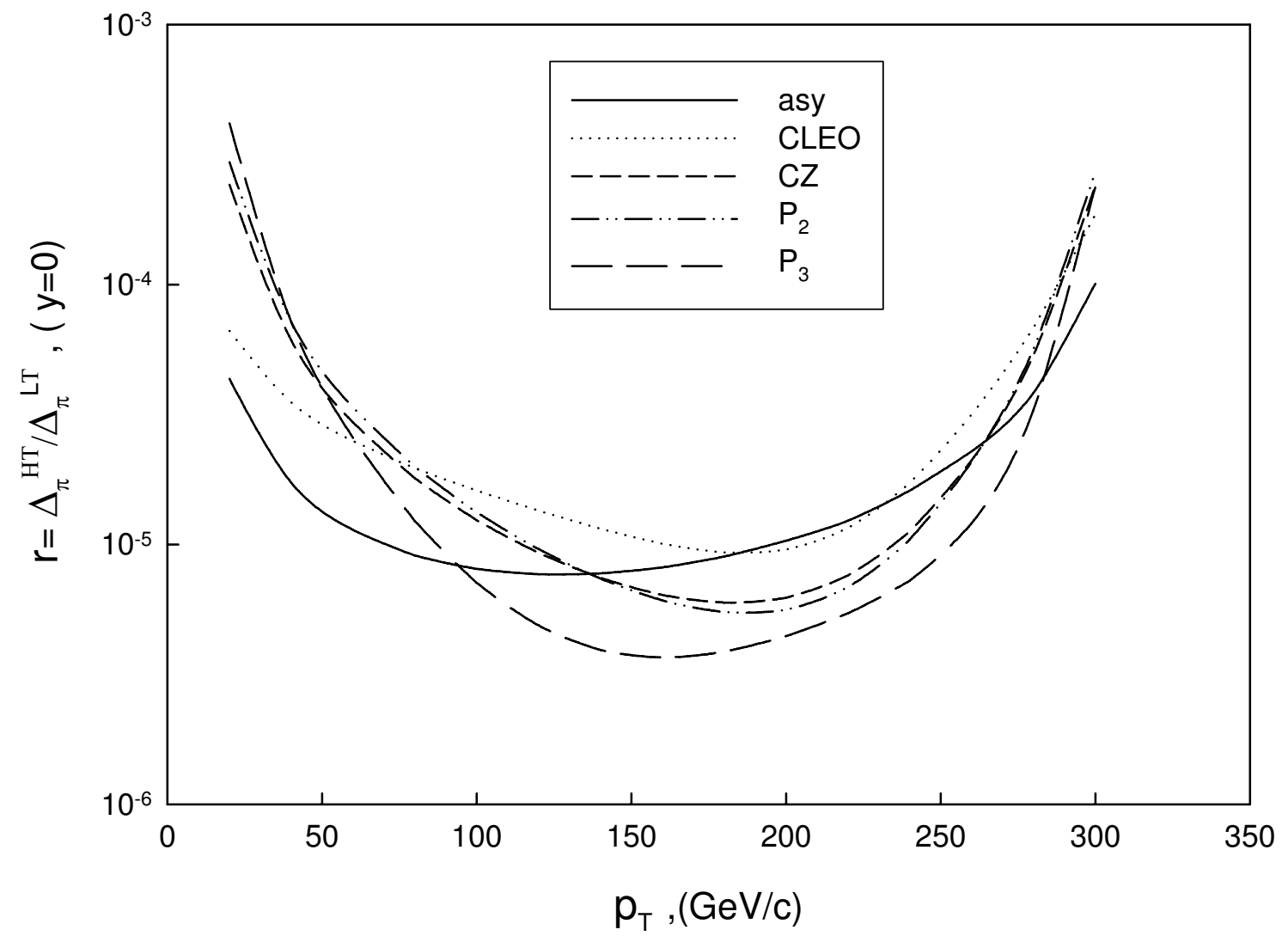

FIG. 16: The ratio $r=\Delta_{\pi}^{H T} / \Delta_{\pi}^{L T}$, where the difference of the leading and the high twist contributions are calculated for the pion rapidity, $y=0$, at the c.m. energy $\sqrt{s}=630 G e V$, as a function of the pion transverse momentum $p_{T}$. 


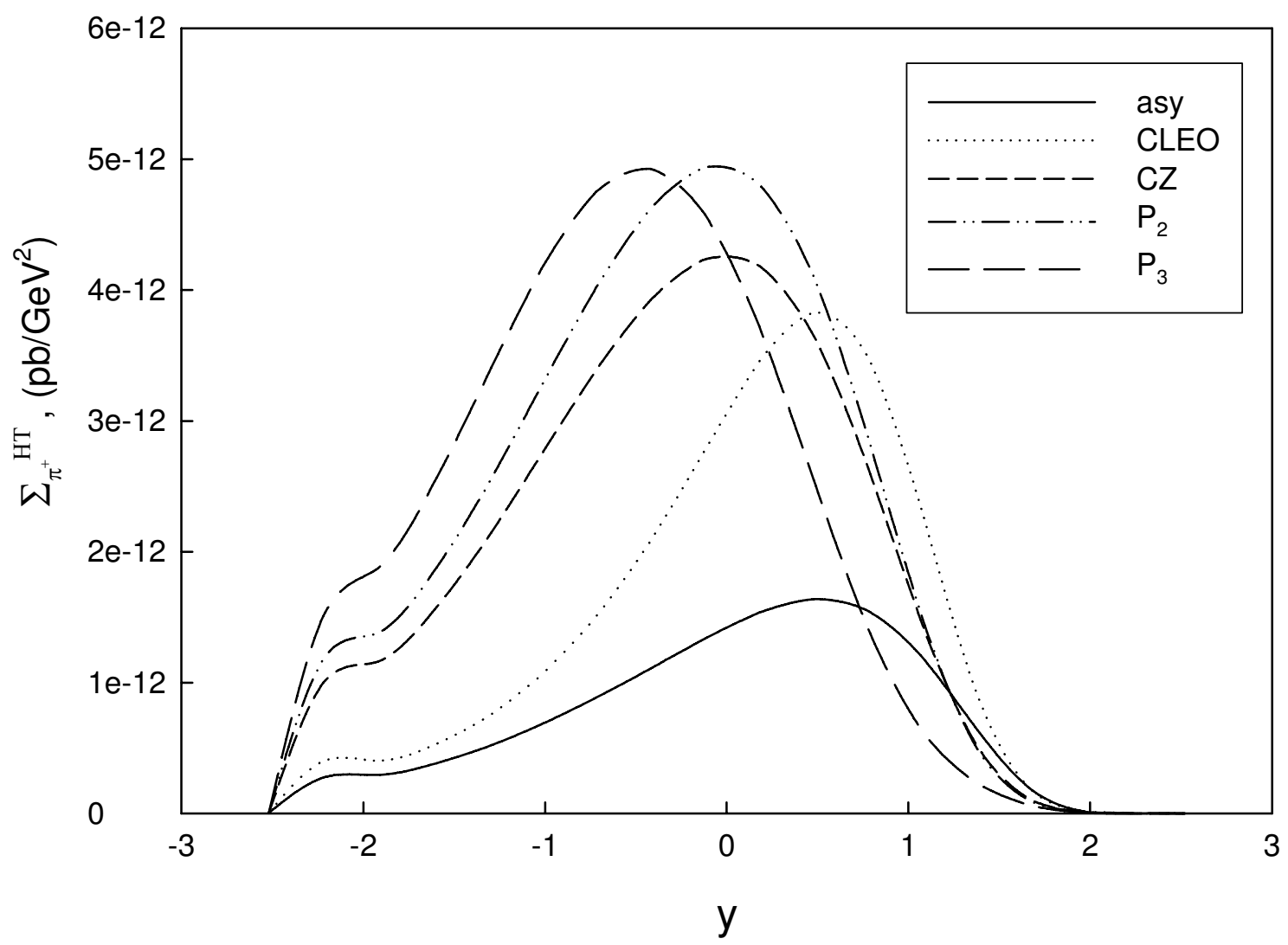

FIG. 17: The high twist $\pi^{+}$production cross sections as a function of the $y$ rapidity of the pion at the transverse momentum of the pion $p_{T}=50 \mathrm{GeV}$, at c.m. energy $\sqrt{s}=630 \mathrm{GeV}$.

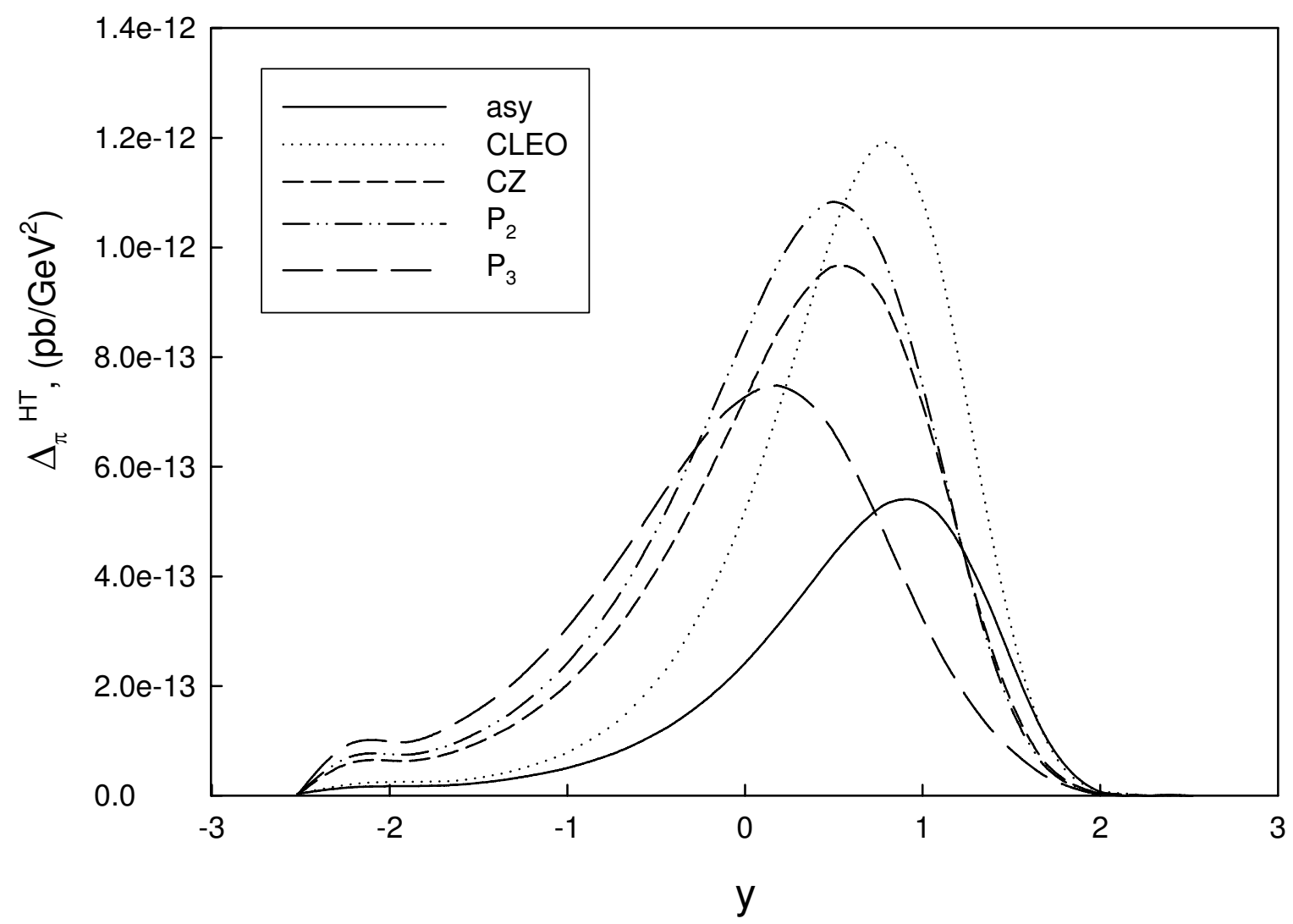

FIG. 18: The difference of the high twist cross section, $\Delta_{\pi}^{H T}=\Sigma_{\pi^{+}}^{H T}-\Sigma_{\pi^{-}}^{H T}$, as a function of the $y$ rapidity of the pion at the transverse momentum of the pion $p_{T}=50 G e V$, at c.m. energy $\sqrt{s}=630 \mathrm{GeV}$. 